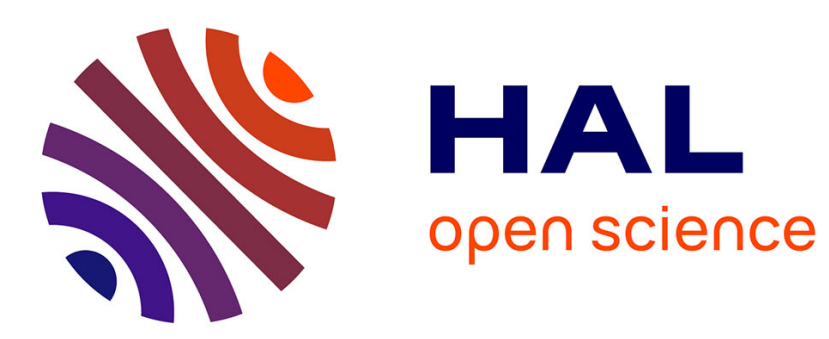

\title{
Do irrelevant commodities matter?
}

Marc Fleurbaey, Koichi Tadenuma

\section{- To cite this version:}

Marc Fleurbaey, Koichi Tadenuma. Do irrelevant commodities matter?. Econometrica, 2007, 75, pp.1143-1174. hal-00246315

\section{HAL Id: hal-00246315 https://hal.science/hal-00246315}

Submitted on 7 Feb 2008

HAL is a multi-disciplinary open access archive for the deposit and dissemination of scientific research documents, whether they are published or not. The documents may come from teaching and research institutions in France or abroad, or from public or private research centers.
L'archive ouverte pluridisciplinaire HAL, est destinée au dépôt et à la diffusion de documents scientifiques de niveau recherche, publiés ou non, émanant des établissements d'enseignement et de recherche français ou étrangers, des laboratoires publics ou privés. 


\title{
Do Irrelevant Commodities Matter?*
}

\author{
Marc Fleurbaey $^{\dagger} \quad$ Koichi Tadenuma ${ }^{\ddagger}$
}

December 2005

Revision: February 2007

\begin{abstract}
We study how to evaluate allocations independently of individual preferences over unavailable commodities. We prove impossibility results suggesting that such evaluations encounter serious difficulties. This is related to the well-known problem of performing international comparisons of standard of living across countries with different consumption goods. We show how possibility results can be retrieved with restrictions on the domain of preferences, on the application of the independence
\end{abstract}

*We thank the Editor, L. Samuelson, and three referees for very helpful comments and suggestions. We also thank J. Bone, R. Bradley, P.A. Chiappori, R. Deb, F. Maniquet, M. Mariotti, H. Moulin, K. Roberts, K. Suzumura, W. Thomson, and P. Young for comments, as well as participants in seminars at CORE, the LSE, Oxford, Columbia, Rice, SMU and Waseda, and in the Asian Decentralization Conference 2005. A first draft of this paper was prepared while K. Tadenuma was visiting the University of Pau. He is grateful for the hospitality and financial support of the University. Financial support from the Ministry of Education, Culture, Sports, Science and Technology of Japan for the 21st Century Center of Excellence Project on the Normative Evaluation and Social Choice of Contemporary Economic Systems is also gratefully acknowledged.

${ }^{\dagger}$ CNRS-CERSES, University Paris-Descartes, LSE and IDEP. Email: marc.fleurbaey@univ-paris5.fr.

${ }_{\ddagger}^{\ddagger}$ Faculty of Economics, Hitotsubashi University, Tokyo. Email: tadenuma@econ.hit-u.ac.jp. 
axiom or on the set of allocations to be ranked. Such restrictions appear more plausible when the objects of evaluation are allocations of composite commodities, characteristics or human functionings rather than ordinary commodities.

Keywords: consumer preferences, social choice, independence of irrelevant alternatives, characteristics, functionings.

Journal of Economic Literature Classification Numbers: D63, D71. 


\section{Introduction}

When a reform of economic policy is considered, welfare economics suggests that it should be evaluated on the basis of its consequences over the population, taking account of individual preferences. It usually goes without saying that the preferences taken into account bear only on the commodities that are available in the options considered. In this paper we explore the implications of this natural restriction, and show that it is far from innocuous.

Restricting attention to individual preferences over available commodities appears to be a matter of practical necessity since very little is known about preferences over unavailable commodities due to lack of observation of choices. Examples can be drawn from space and time heterogeneity of commodities. Space differences are most striking across distant countries. Japanese preferences over the various kinds of sheep cheese from different valleys in the French Pyrenees are hardly known, and it is no wonder that reforms in Japan are not evaluated on the basis of the local population's preferences over these varieties of cheese. Similarly, French preferences over varieties of sake are difficult to guess, and reforms in France are never discussed by reference to preferences over sake. Changes of commodities in time also give obvious examples. Modern preferences over the use of pigeon post are unknown, and we never hear about preferences concerning pigeon post nowadays even in the discussion of reforms of the postal service.

Heterogeneity of commodities is also the source of well-known problems in comparisons of living standards across space and time. The fact that different countries have different consumption goods in their local markets makes it difficult to compare prices, and the computation of purchasing power parities has to rely on gross approximations. For instance, the few items that are common in two countries are used to compute indexes of relative prices for broad categories of goods. A similar problem occurs in time series for 
the evaluation of growth over long periods of time. In this paper, we do not primarily focus on comparisons across different populations, but on the evaluation of different allocations for a given population. The two exercises are nonetheless related since the evaluation of an allocation for a population usually involves comparing the living standards of its members. The issue of comparisons will therefore also be addressed here.

In this paper, we examine the possibility of making social evaluations of allocations on the sole basis of individual preferences over available commodities. More precisely, we introduce the condition of Independence of Irrelevant Commodities (IIC), stating that when two allocations have zero quantities of some commodities, the social ranking of the two allocations should be independent of individual preferences over these commodities. Our framework is borrowed from the theory of social choice in economic environments, surveyed, for instance, by Le Breton (1997) and Le Breton and Weymark (2000).

IIC is similar to the well-known condition due to Arrow (1951), Independence of Irrelevant Alternatives (IIA), but it turns out to be much weaker and, we believe, much less controversial. Let us briefly explain why. IIA states that the ranking of two allocations should depend only on individual preferences over these two allocations. This requirement is often implausible, especially in economic environments. For instance, suppose that half of the population prefers allocation $x$ and half of the population prefers allocation $y$. How can we rank $x$ and $y$ with this limited information? Under IIA, we may not explore whether any one of these allocations is Pareto-efficient, envy-free, ${ }^{1}$ egalitarian-equivalent, ${ }^{2}$ or a Walrasian equilibrium with equal budgets because such exploration requires checking how bundles in $x$ or $y$ are ranked in individual preferences with respect to other bundles. In contrast, IIC allows us to use all the information about individual preferences

\footnotetext{
${ }^{1}$ An allocation is envy-free (Foley 1967, Kolm 1972) if no agent prefers another's bundle to his own.

${ }^{2}$ An allocation is egalitarian-equivalent (Pazner and Schmeidler 1978) if there exists a bundle $x_{0}$, proportional to total consumption, such that every agent is indifferent between his bundle and $x_{0}$.
} 
over available commodities, and this is sufficient in order to assess whether any of these allocations is Pareto-efficient, envy-free, and so on.

We may even claim that our condition is actually more faithful to Arrow's initial vision. As a defense of his condition for applications to the evaluation of resource allocations, Arrow wrote (1950, p. 346; see also 1951, p. 73):

'Suppose that there are just two commodities, bread and wine. A distribution, deemed equitable by all, is arranged, with the wine-lovers getting more wine and less bread than the abstainers. Suppose now that all the wine is destroyed. Are the wine-lovers entitled, because of that fact, to more than an equal share of bread? The answer is, of course, a value judgment. My own feeling is that tastes for unattainable alternatives should have nothing to do with the decision among the attainable ones; desires in conflict with reality are not entitled to consideration.'

This example suggests that when wine is not available, preferences over wine should be disregarded. This is exactly what our IIC states, whereas Arrow's IIA requires much more. Besides, Arrow's mention of "equal shares" is in fact an introduction of fairness considerations which cannot be accommodated within the informational straitjacket of Arrow's condition.

We do not consider Arrow's impossibility theorem to be a serious obstacle to social choice in economic environments because IIA imposes too severe a restriction on information about individual preferences. Unfortunately, as we show, our condition still entails a similar impossibility result even though it is much weaker than IIA. Combined with a Pareto condition embodying the respect for unanimous individual preferences, it implies that the social preference rule must be dictatorial (i.e. social preferences must always obey one particular agent's strict preferences) in a large set of cases which is not exactly 
as large as in Arrow's theorem but still quite substantial. We consider this result to be much more disturbing than Arrow's theorem. However, we also examine here how this difficulty can be tackled in order to obtain positive solutions.

Our work is related to the small part of the social choice literature which has been critical of Arrow's independence condition and has examined how to construct fair social preferences when this condition is relaxed. Mayston (1974, 1982), Pazner (1979), Fleurbaey and Maniquet (2005), Fleurbaey, Suzumura and Tadenuma (2005a,b) studied how to rely on individual indifference surfaces in order to construct "fair" social preferences. ${ }^{3}$ In this literature, the main independence condition, among the less restrictive, allows social evaluations to use information about the whole indifference surface of every individual at the contemplated allocations in order to rank these allocations. As shown by these authors, nice social preference rules can be constructed under this independence condition. Our IIC is neither weaker nor stronger than this. It allows us to retain information about the whole preference maps in the subspace of consumed commodities while discarding the rest of the indifference surfaces. More closely related to our condition of independence is Donaldson and Roemer's (1987) consistency condition, which essentially requires social evaluations to disregard commodities with identical quantities in the allocations to be compared. They also derive an impossibility theorem, but their framework is different since it involves utility functions, whereas we consider only ordinal non-comparable preferences. Moreover, their consistency condition is somehow stronger than ours. We only require social evaluation to disregard individual preferences over commodities with zero

\footnotetext{
${ }^{3}$ There is much more extensive literature, initiated by Sen (1970), which also rejects Arrow's independence but proposes to construct social preferences on the basis of interpersonally comparable utility information instead of additional information about ordinal non-comparable preferences. In this paper we stick to Arrow's (1951) purely ordinal approach, which can be defended on various grounds, as discussed e.g. in Fleurbaey (2003).
} 
quantities, but require nothing concerning commodities with identical positive quantities.

The rest of the paper is organized as follows. Section 2 presents a simple example conveying the main intuition for our results. Sections 3 and 4 introduce the formal framework and the main notions. Section 5 states and proves the main result. Section 6 addresses the related issues of subpopulations consuming different commodities and of comparisons of living standards, by introducing a variant of IIC. Since impossibility results should not stop us from seeking to make good social evaluations, Section 7 examines the best strategies available in order to obtain social preferences that are not only nondictatorial but also satisfy an anonymity requirement.

\section{A tale of two commodities}

With a simple example, this section shows why it may entail a difficulty to require social evaluations to disregard individual preferences about unavailable commodities. On Robinson and Friday's island, two commodities may be available, bread and wine. Individual preferences are assumed to be strictly monotonic.

Consider the four allocations described in the following table.

\begin{tabular}{|c|c|c|c|c|}
\cline { 2 - 5 } \multicolumn{1}{c|}{} & \multicolumn{2}{c|}{ Robinson } & \multicolumn{2}{c|}{ Friday } \\
\cline { 2 - 5 } \multicolumn{1}{c|}{} & bread & wine & bread & wine \\
\hline$x$ & 3 & 0 & 7 & 0 \\
\hline$y$ & 7 & 0 & 3 & 0 \\
\hline$z$ & 0 & 4 & 0 & 8 \\
\hline$w$ & 0 & 8 & 0 & 4 \\
\hline
\end{tabular}

If we rank all allocations, either allocation $x$ is better than allocation $y$, or conversely, or they are indifferent. Suppose first that $x$ is better than $y$ for some preference profile 
$R_{N}$. We now show that, under this assumption, along with the Pareto principle and the independence requirement that social evaluations disregard individual preferences about unavailable commodities, for any preference profile $R_{N}^{\prime}$, and for any two allocations $x^{\prime}$ and $y^{\prime}$ containing only bread such that Friday gets more at $x^{\prime}$ than at $y^{\prime}$ whereas Robinson consumes less at $x^{\prime}$ than at $y^{\prime}, x^{\prime}$ is better than $y^{\prime}$.

To prove the claim, consider the preference profile $R_{N}^{*}$ such that Robinson's and Friday's preferences rank the above four allocations as follows (in decreasing order):

\begin{tabular}{|c|c|}
\hline Robinson & Friday \\
\hline$y$ & $z$ \\
\hline$w$ & $x$ \\
\hline$z$ & $y$ \\
\hline$x$ & $w$ \\
\hline
\end{tabular}

Since individual preferences are strictly monotonic, $R_{N}^{*}$ agrees with $R_{N}$ on the space of bread-only allocations. Hence, under our independence requirement and the assumption that $x$ is better than $y$ for $R_{N}$, the same must hold for $R_{N}^{*}$. We also see that both individuals prefer $z$ to $x$ and $y$ to $w$ at $R_{N}^{*}$. If we respect unanimous preferences (the Pareto principle), then $z$ is better than $x$ and $y$ is better than $w$ for $R_{N}^{*}$. By transitivity we conclude that $z$ is better than $w$ for $R_{N}^{*}$.

Now choose any preference profile $R_{N}^{\prime}$ and any two bread-only allocations $x^{\prime}$ and $y^{\prime}$ such that $x_{R}^{\prime}<y_{R}^{\prime}$ and $x_{F}^{\prime}>y_{F}^{\prime}$ where $x_{R}^{\prime}$ (resp., $x_{F}^{\prime}$ ) denotes Robinson's (resp., Friday's) consumption of bread at $x^{\prime}$, and so on.

Consider the preference profile $R_{N}^{*}$ such that: 


\begin{tabular}{|c|c|}
\hline Robinson & Friday \\
\hline$w$ & $x^{\prime}$ \\
\hline$y^{\prime}$ & $z$ \\
\hline$x^{\prime}$ & $w$ \\
\hline$z$ & $y^{\prime}$ \\
\hline
\end{tabular}

Note that $R_{N}^{*}$ agrees with $R_{N}^{*}$ on the space of wine-only allocations, and it also agrees with $R_{N}^{\prime}$ on the space of bread-only allocations. Since $z$ is better than $w$ for $R_{N}^{*}$, our independence condition implies that $z$ is better than $w$ for $R_{N}^{*}$. Together with the Pareto principle and transitivity, we conclude that $x^{\prime}$ is better than $y^{\prime}$ for $R_{N}^{*}$. Applying our independence condition to $R_{N}^{\prime *}$ and $R_{N}^{\prime}$, the same holds for $R_{N}^{\prime}$. Since $R_{N}^{\prime}$ was chosen arbitrarily, our claim has been proved.

For any bread-only allocations $x^{\prime}$ and $y^{\prime}$, if $x_{R}^{\prime}>y_{R}^{\prime}$ and $x_{F}^{\prime}>y_{F}^{\prime}$, then the Pareto principle implies that $x^{\prime}$ is better than $y^{\prime}$. If $x_{R}^{\prime}=y_{R}^{\prime}$ and $x_{F}^{\prime}>y_{F}^{\prime}$, then one can consider $y^{\prime \prime}$ such that $y_{R}^{\prime \prime}>x_{R}^{\prime}=y_{R}^{\prime}$ and $x_{F}^{\prime}>y_{F}^{\prime \prime}>y_{F}^{\prime}$. Then, $y^{\prime \prime}$ is better than $y^{\prime}$ from the Pareto principle, and by the above claim, $x^{\prime}$ is better than $y^{\prime \prime}$. Hence, $x^{\prime}$ is better than $y^{\prime}$.

We have therefore shown that for any bread-only allocations $x^{\prime}$ and $y^{\prime}$, if Friday prefers $x^{\prime}$ to $y^{\prime}$ (which is equivalent to $x_{F}^{\prime}>y_{F}^{\prime}$ ), then $x^{\prime}$ is better than $y^{\prime}$. That is, Friday is a "dictator" over all bread-only allocations.

A similar argument starting with the above observation that $z$ is better than $w$ for $R_{N}^{*}$ will show that Friday is a "dictator" over all wine-only allocations. It can also be shown that Friday's dictatorship extends over comparisons between bread-only allocations and wine-only ones.

By symmetry, if we assume that $y$ is better than $x$ for some preference profile in the first place, then we obtain the conclusion that Robinson is a dictator over all onecommodity allocations. If we assume that $x$ and $y$ are indifferent, then by slightly improv- 
ing the bundles in $x$ for both agents, we would obtain a similar case as when $x$ is better than $y$, and would therefore show again that Friday is a dictator over one-commodity allocations. But by slightly improving the bundles in $y$ we would obtain that Robinson is also a dictator over one-commodity allocations. Since these two conclusions are incompatible, it is in fact impossible here for the social evaluation to be indifferent between $x$ and $y$.

Let us come back to the assumption that $x$ is better than $y$ for at least one preference profile. As we have shown, Friday then becomes a dictator over all one-commodity allocations. Is Friday a dictator over all allocations, not just those with only one commodity? Consider any pair of two-commodity allocations $a$ and $b$. Suppose, for instance, that Friday prefers $a$ to $b$. If Robinson prefers $a$ to $b$ as well, we conclude from the Pareto principle that $a$ is better than $b$. What if Robinson prefers $b$ to $a$ ? Suppose that there is a pair of bread-only allocations, $x^{\prime}$ and $y^{\prime}$, such that individual preferences are as follows:

\begin{tabular}{|c|c|}
\hline Robinson & Friday \\
\hline$y^{\prime}$ & $a$ \\
\hline$b$ & $x^{\prime}$ \\
\hline$a$ & $y^{\prime}$ \\
\hline$x^{\prime}$ & $b$ \\
\hline
\end{tabular}

Since Friday is a dictator for one-commodity allocations, the social ranking of $x^{\prime}$ and $y^{\prime}$ conforms to Friday's preferences. Together with the Pareto principle and transitivity, we can conclude that $a$ is better than $b$. It seems that Friday is a dictator for all allocations, including two-commodity allocations.

This conclusion, however, would be hasty. Suppose that Robinson prefers the twocommodity bundle $b$ to all bundles containing only one commodity. Then it is impossible to find a one-commodity allocation $y^{\prime}$ better than $b$ for him, and the above reasoning 
fails. It is then indeed possible to rank $b$ above $a$, against Friday's preference. Friday's dictatorship need not extend to all allocations. This appears to depend on whether agents can reach arbitrarily high indifference curves with a reduced number of commodities.

This simple example provides intuition for the main elements of our analysis: 1) the condition of Independence of Irrelevant Commodities may still entail dictatorship, especially for allocations with missing commodities; 2) non-dictatorial social evaluation is however possible in some cases; 3) a key fact is whether individuals, according to their own preferences, can find better bundles containing fewer commodities.

\section{Framework}

Let $L:=\{1, \ldots, \ell\}$ be the set of commodities, and $N:=\{1, \ldots, n\}$ the set of agents, where $2 \leq \ell<\infty$ and $2 \leq n<\infty$. Denoting $\mathbb{R}_{+}$the set of all non-negative real numbers, $\mathbb{R}_{+}^{\ell}$ is the set of all consumption bundles. Agent $i$ 's consumption bundle is a vector $x_{i}:=\left(x_{i 1}, \ldots, x_{i \ell}\right) \in \mathbb{R}_{+}^{\ell}$. An allocation is a vector $x:=\left(x_{1}, \ldots, x_{n}\right) \in \mathbb{R}_{+}^{\ell n}$. The set of all allocations is $\mathbb{R}_{+}^{\ell n}$. The set of allocations such that no individual bundle $x_{i}$ is equal to the zero vector is denoted $X$, i.e., $X:=\left(\mathbb{R}_{+}^{\ell} \backslash\{0\}\right)^{n}$.

In order to study allocations in which some of the $\ell$ commodities are absent, we introduce the following notion of a subspace. For each $K \subseteq L$, define $\mathbb{R}_{+}^{K} \subseteq \mathbb{R}_{+}^{\ell}$ by

$$
\mathbb{R}_{+}^{K}:=\left\{q \in \mathbb{R}_{+}^{\ell} \mid \forall k \in L \backslash K, q_{k}=0\right\}
$$

Notice that $\mathbb{R}_{+}^{K}$ is a subset of $\mathbb{R}_{+}^{\ell}$, so that $q \in \mathbb{R}_{+}^{K}$ is a full vector with $\ell$ components, some of which are simply null. Let $\mathbb{R}_{++}^{K}$ denote the subset of $\mathbb{R}_{+}^{K}$ containing bundles such that all commodities in $K$ are in positive quantity.

An ordering is a reflexive and transitive binary relation. For each $i \in N$, agent $i$ 's preference relation is a complete ordering $R_{i}$ on $\mathbb{R}_{+}^{\ell}$, that is, on $i$ 's personal bundles. 
This means that, as is standard in microeconomics, we restrict attention to self-centered preferences. The strict preference relation and the indifference relation associated to $R_{i}$ are denoted $P_{i}$ and $I_{i}$, respectively. Let $\mathcal{R}$ be the set of continuous, convex, and strictly $^{4}$ monotonic preference relations. A profile of preference relations is a list $R_{N}:=$ $\left(R_{1}, \ldots, R_{n}\right) \in \mathcal{R}^{n}$

A social ordering function is a mapping $\Psi$ defined on $\mathcal{R}^{n}$, such that for all $R_{N} \in \mathcal{R}^{n}$, $\Psi\left(R_{N}\right)$ is a complete ordering on the set of all allocations $\mathbb{R}_{+}^{\ell n} \cdot \Psi\left(R_{N}\right)$ is interpreted as the social ordering of all allocations when agents' preferences are $R_{N}$. We simply denote by $R$ (with no subscript) the social ordering $\Psi\left(R_{N}\right)$, by $R^{\prime}$ the social ordering $\Psi\left(R_{N}^{\prime}\right)$, and so on, when no confusion may arise.

We will repeatedly require the social ordering function to obey the Weak Pareto condition saying that unanimous strict preference must be respected. This is a very basic condition of respect of individual preferences. It is especially compelling when dealing, as here, with self-centered preferences. It then means that individuals are sovereign over their personal consumption.

Weak Pareto: $\forall R_{N} \in \mathcal{R}^{n}, \forall x, y \in \mathbb{R}_{+}^{\ell n}$, if $\forall i \in N, x_{i} P_{i} y_{i}$, then $x P y$.

We also need to define the notion of dictatorship, and we propose a definition that encompasses situations where the dictator dictates only in a subset of cases. Let $\mathcal{D}$ be a subset of $\mathcal{R}^{n}$ and $Y$ a subset of $\mathbb{R}_{+}^{\ell n}$. We say that an agent $i_{0} \in N$ is a dictator over $(\mathcal{D}, Y)$ for the social ordering function $\Psi$ if for all $R_{N} \in \mathcal{D}$, and all $x, y \in Y, x_{i_{0}} P_{i_{0}} y_{i_{0}}$ implies $x P y$. When there is such an agent, the social ordering function $\Psi$ is said to be dictatorial over $(\mathcal{D}, Y)$. Clearly, since a dictator over $(\mathcal{D}, Y)$ is also a dictator over any $\left(\mathcal{D}^{\prime}, Y^{\prime}\right)$ with $\mathcal{D}^{\prime} \subseteq \mathcal{D}$ and $Y^{\prime} \subseteq Y$, the larger the sets $\mathcal{D}$ and $Y$, the greater the extent of dictatorship.

\footnotetext{
${ }^{4}$ For a discussion of the role of strict monotonicity in our analysis, see the appendix.
} 


\section{Sufficient and dispensable commodities}

The example in Section 2 tells us that it is important to see whether an agent can find better bundles with fewer commodities. In this section we introduce the corresponding definitions.

Let $K \subseteq L$ be a set of commodities, and let $i \in N$ and $R_{i} \in \mathcal{R}$ be given. We call $K$ a sufficient set for $R_{i}$ if all bundles in $\mathbb{R}_{+}^{\ell}$ can be surpassed in preference by bundles in $\mathbb{R}_{+}^{K}:$

$$
\forall x_{i} \in \mathbb{R}_{+}^{\ell}, \exists y_{i} \in \mathbb{R}_{+}^{K}, y_{i} R_{i} x_{i}
$$

Otherwise, it is called an insufficient set. In the latter case, one can say that satisfaction is "bounded" in $\mathbb{R}_{+}^{K}$, in the sense that there are some indifference surfaces for $R_{i}$ that cannot be surpassed in preference by any bundle of $\mathbb{R}_{+}^{K}$. For instance, with only water, bread and log cabins, satisfaction may be bounded, so that this set of three commodities is insufficient. In contrast, with all the sorts of commodities typically available in a given country, one can obtain every possible level of satisfaction, so that this forms a sufficient set.

When $K$ is a sufficient set, we say that the complement set $M=L \backslash K$ is a dispensable set. Indeed, this means that satisfaction is not bounded in the absence of the commodities in $M$. Conversely, when satisfaction is bounded in the absence of commodities in $M, M$ will be called an indispensable set. For instance, apple fritters and skating may be dispensable for some preferences, whereas water and newspapers may be indispensable for these same preferences. The following table summarizes these notions.

\begin{tabular}{c|c} 
Satisfaction is bounded in $\mathbb{R}_{+}^{K}$ & Satisfaction is not bounded in $\mathbb{R}_{+}^{K}$ \\
\hline$K$ is insufficient & $K$ is sufficient \\
$L \backslash K$ is indispensable & $L \backslash K$ is dispensable
\end{tabular}


If $K$ is sufficient (resp., indispensable) for $R_{i}$, then any $K^{\prime} \supseteq K$ is also sufficient (resp., indispensable) for $R_{i}$. If $K$ is insufficient (resp., dispensable) for $R_{i}$, then any $K^{\prime} \subseteq K$ is also insufficient (resp., dispensable) for $R_{i}$. The set $L$ is sufficient and indispensable for all preference relations in $\mathcal{R}$. Notice that the complement of a sufficient (resp., dispensable) set need not be insufficient (resp., indispensable).

These notions may be of some interest for consumer theory. This theory contains the notion of an essential commodity, that is, a commodity such that without it, utility (or production when it is about a production function) is at the smallest level. Though the notions introduced above deal with possibly high levels of satisfaction, there is an obvious link: An essential commodity is necessarily indispensable. A commodity that is not essential, however, may still be indispensable.

\section{Independence of Irrelevant Commodities}

As explained in the introduction, we require the social ranking of two allocations to depend only on individual preferences for commodities that are available in these allocations. Formally, our condition states that a change of individual preferences for unavailable commodities should not alter the social ranking. Or, equivalently, if two preference profiles agree on all of $\left(\mathbb{R}_{+}^{K}\right)^{n}$, then for any $x, y \in\left(\mathbb{R}_{+}^{K}\right)^{n}$, the social rankings for these profiles agree on $\{x, y\}$.

Independence of Irrelevant Commodities (IIC): $\forall R_{N}, R_{N}^{\prime} \in \mathcal{R}^{n}, \forall x, y \in \mathbb{R}_{+}^{\ell n}$, if $\exists K \subseteq L$ such that $x, y \in\left(\mathbb{R}_{+}^{K}\right)^{n}$ and $\forall i \in N, R_{i}$ and $R_{i}^{\prime}$ agree on $\mathbb{R}_{+}^{K}$, then $R$ and $R^{\prime}$ agree on $\{x, y\}$.

Our IIC condition is logically weaker than Arrow's IIA condition, which requires that for any given pair of allocations, a change of individual preferences about a third 
allocation should not alter the social ranking between the given two allocations.

Independence of Irrelevant Alternatives (IIA): $\forall R_{N}, R_{N}^{\prime} \in \mathcal{R}^{n}, \forall x, y \in \mathbb{R}_{+}^{\ell n}$, if $\forall i \in N, R_{i}$ and $R_{i}^{\prime}$ agree on $\{x, y\}$, then $R$ and $R^{\prime}$ agree on $\{x, y\}$.

The informational basis on which the desirability of allocations is assessed is much expanded with IIC, compared to IIA. As we mentioned in the introduction, under IIC we may examine whether an allocation is Pareto-efficient, envy-free, egalitarian-equivalent, or Walrasian with equal budgets, whereas under IIA such examination is impossible. Moreover, under IIC, we may measure an "intensity" of individual preferences for $x$ over $y$ in a similar fashion as with the Borda rule in the voting context, whereas this cannot be done under IIA. For instance, consider two allocations $x, y \in\left(\mathbb{R}_{+}^{K}\right)^{n}$. Take a reference bundle $x_{0} \in \mathbb{R}_{++}^{K}$, and define

$$
v_{R_{i}}\left(x_{i} ; x_{0}\right)=\min \left\{\lambda \in \mathbb{R}_{+} \mid \lambda x_{0} R_{i} x_{i}\right\}
$$

Then, the difference $v_{R_{i}}\left(x_{i} ; x_{0}\right)-v_{R_{i}}\left(y_{i} ; x_{0}\right)$ may be interpreted as a kind of "intensity" of individual preferences for $x$ over $y$, and such information may be used in order to rank $x$ and $y$, while obeying IIC.

Arrow's theorem, adapted to this framework, states that a social ordering function satisfying Weak Pareto and IIA must be dictatorial over $\left(\mathcal{R}^{n}, X\right) .{ }^{5}$ As we show below, this impossibility no longer holds when IIA is replaced by IIC, but we still obtain dictatorship in a substantial set of cases, corresponding to certain subsets of profiles of preferences and of allocations, which we now define.

\footnotetext{
${ }^{5}$ Arrow's (1950) initial presentation of his theorem was already dealing with our economic framework, but the rigorous proof of his theorem for economic models owes much to Kalai et al. (1979) for the case of public goods, and Bordes and Le Breton (1989) for that of private goods. As first noticed by Border (1983), dictatorship is obtained for $X$ only, because, by monotonicity of preferences, the zero bundle is never part of a free triple (see the appendix for a definition of this notion).
} 
Let $\mathcal{D}^{-} \subseteq \mathcal{R}^{n}$ denote the subset of profiles such that there is a proper subset $K \subsetneq L$ that is sufficient (and its non-empty complement is dispensable) for all $i \in N$. Formally:

$$
\begin{aligned}
\mathcal{D}^{-} & :=\left\{R_{N} \in \mathcal{R}^{n} \mid \exists K \subseteq L, K \neq L, \forall i \in N, K \text { is sufficient for } R_{i}\right\} \\
& =\left\{R_{N} \in \mathcal{R}^{n} \mid \exists M \subseteq L, M \neq \emptyset, \forall i \in N, M \text { is dispensable for } R_{i}\right\} .
\end{aligned}
$$

Let $\bar{X}$ be the subset of $X$ such that at least one commodity is absent from the allocation, i.e.

$$
\bar{X}:=\left\{x \in X \mid \sum_{i \in N} x_{i} \notin \mathbb{R}_{++}^{\ell}\right\} .
$$

Our main result can now be stated. The proof is in the appendix.

Theorem 1 If a social ordering function satisfies Weak Pareto and Independence of Irrelevant Commodities, then there is an agent who is a dictator for this social ordering function over $\left(\mathcal{D}^{-}, X\right)$ and over $\left(\mathcal{R}^{n}, \bar{X}\right)$.

Compared with dictatorship over $\left(\mathcal{R}^{n}, X\right)$ in Arrow's theorem, we see that dictatorship still prevails when restricting attention either to the set of preference profiles $\mathcal{D}^{-}$or to the set of allocations $\bar{X}$.

In the appendix, we also show that the above theorem is "tight" in the sense that $\mathcal{D}^{-}$ is a maximal domain of dictatorship for $X$, and that $\bar{X}$ is a maximal scope of dictatorship for $\mathcal{R}^{n}$. More precisely, we exhibit a social ordering function that is not dictatorial over $\left(\left\{R_{N}\right\}, X\right)$ for every $R_{N} \in \mathcal{D}^{+}:=\mathcal{R}^{n} \backslash \mathcal{D}^{-}$, and another social ordering function that is not dictatorial over $\left(\mathcal{R}^{n},\{x, y\}\right)$ for every pair $\{x, y\} \subseteq X \backslash \bar{X}$ except those such that $x_{i}>y_{i}$ for all $i \in N$ or $x_{i}<y_{i}$ for all $i \in N,{ }^{6}$ and those such that $x_{i}=y_{i}$ for some $i \in N{ }^{7}$.

\footnotetext{
${ }^{6}$ Vector inequalities are denoted $\gg,>$, and $\geq$.

${ }^{7}$ When $x_{i}>y_{i}$ for all $i \in N$, monotonicity of preferences and Weak Pareto imply $x P y$ for all profiles, and since all agents agree with this ranking, every agent is formally a dictator over $\left(\mathcal{R}^{n},\{x, y\}\right)$. When $x_{i}=y_{i}$, agent $i$ is a dictator over $\left(\mathcal{R}^{n},\{x, y\}\right)$ because the implication $x_{i} P_{i} y_{i} \Rightarrow x P y$ is vacuously true.
} 
Whether we derive a negative or a positive implication from these results depends on how the concept of a commodity is defined. In Debreu (1959, p. 24), it is defined

'by a specification of all its physical characteristics, of its availability date, and of its availability location. As soon as one of these three factors changes, a different commodity results.'

If we consider individual preferences over commodities defined in this way, then it is clear that almost every commodity is dispensable for every individual, so that $\mathcal{D}^{-}$is the appropriate domain of preferences. It is also obvious that there are commodities that no individual in a particular country or period can consume (e.g., people staying in Japan can never consume bread available in France) in which case $\bar{X}$ is the relevant range of allocations. Hence, Arrow's impossibility remains valid with the weaker IIC axiom in this context.

However, if we consider composite commodities, then it becomes less likely that all individuals have the same dispensable commodities, and the likelihood depends on how broadly we define composite commodities. For instance, various kinds of fruit may be dispensable whereas "fruit" may not be. When we define composite commodities in a sufficiently broad sense, we are no longer trapped in dictatorship.

Thus, the above results delineate the borderline in model specification which divides possibility and impossibility of non-dictatorial social choice.

These results, however, are not the end of our investigation. The examples of social ordering functions that are used to check tightness of Theorem 1 are not appealing since they are still dictatorial over substantial subsets of allocations or of preference profiles, ${ }^{8}$

\footnotetext{
${ }^{8}$ More precisely, for any given $R_{N} \in \mathcal{D}^{+}$, dictatorship over $\left(\left\{R_{N}\right\}, \bar{X}\right)$ extends to all allocations in $X \backslash \bar{X}$ that are ranked below some allocations in $\bar{X}$ by all agents (but does not extend to all allocations in $X \backslash \bar{X})$. Similarly, for any given $\{x, y\} \subseteq X \backslash \bar{X}$, dictatorship over $\left(\mathcal{D}^{-},\{x, y\}\right)$ extends to all preference
} 
and hence do not satisfy an anonymity requirement. The construction of anonymous social ordering functions will be investigated in the final section.

\section{Heterogeneous subpopulations}

In the previous section, we focused on the difficulty of evaluating allocations in a country or at a particular period that arises from the fact that not all conceivable commodities are available in the country or at the period. In this section, we consider difficulties due to the fact that different subpopulations may consume different commodities. This issue becomes especially relevant if we want to evaluate "global" allocations in the world or growth paths over several generations. For instance, the evaluation of world allocations has to be done with Japanese preferences over Japanese commodities, French preferences over French commodities, and so on.

This issue is also connected to the problem of comparing living standards across space or time. The comparison of living standards across subpopulations is not the same exercise as ranking allocations for the global population, but the latter does involve comparing the situation of subpopulations. Suppose that we are interested in the comparison of France and Japan. A social evaluation of the world allocations will normally provide us with data for such comparison. For instance, consider the social ordering function introduced by Pazner and Schmeidler (1978) and Pazner (1979), based on the notion of egalitarianequivalence. Given a fixed reference bundle $x_{0} \in \mathbb{R}_{+}^{\ell}$, this social ordering function is defined as follows: $x R y$ if and only

$$
\min _{i \in N} v_{R_{i}}\left(x_{i} ; x_{0}\right) \geq \min _{i \in N} v_{R_{i}}\left(y_{i} ; x_{0}\right)
$$

$\overline{\text { profiles } R_{N} \in \mathcal{D}^{+} \text {such that both } x \text { and } y}$ are ranked below some allocations in $\bar{X}$ by all agents (but does not extend to all profiles in $\mathcal{D}^{+}$). See the appendix. 
where $v_{R_{i}}\left(\cdot ; x_{0}\right)$ is defined as in Section 5 . This amounts to comparing individual situations by the minimal fraction of $x_{0}$ that individuals would be willing to substitute for $x_{i}$, and applying the maximin criterion to the vector of such individual measures. We can then also compare the distribution of these indexes in two populations. This is only an example, ${ }^{9}$ but it shows how comparisons are linked to general criteria of social evaluation.

Our IIC condition does not capture the problem raised in this section because every commodity is consumed by someone in the world (or by some generation in the intertemporal context). In order to formalize this issue, we examine the idea of applying the independence requirement in a decentralized way: When two allocations give a zero quantity of some commodity to an individual, the decentralized version of IIC says that the social evaluation should not depend on his preferences for this commodity. For simplicity, we formalize the condition in terms of individuals rather than subpopulations, but the axiom and the analysis can be adapted to subgroups as we will see later.

Individual Independence of Irrelevant Commodities (IIIC): $\forall R_{N}, R_{N}^{\prime} \in \mathcal{R}^{n}$, $\forall x, y \in \mathbb{R}_{+}^{\ell n}$, if $\forall i \in N, \exists K_{i} \subseteq L$ such that $x_{i}, y_{i} \in \mathbb{R}_{+}^{K_{i}}$ and $R_{i}$ and $R_{i}^{\prime}$ agree on $\mathbb{R}_{+}^{K_{i}}$, then $R$ and $R^{\prime}$ agree on $\{x, y\}$.

IIIC is logically stronger than IIC because if the hypothesis of IIC holds, then that of IIIC holds, but not vice versa. We next show that with IIIC, dictatorship extends to a larger preference domain and to a larger set of allocations than with IIC. On this preference domain, every agent has her own sufficient set of commodities with which her satisfaction is not bounded. Formally, we define the new domain of preference profiles as

\footnotetext{
${ }^{9}$ This social ordering function has been axiomatically characterized by Fleurbaey (2005) and Tadenuma (2005). These axiomatic studies may provide some justifications for comparing living standards by these indexes.
} 
follows.

$$
\mathcal{D}^{-*}:=\left\{R_{N} \in \mathcal{R}^{n} \mid \forall i \in N, \exists K \subseteq L, K \neq L, K \text { is sufficient for } R_{i}\right\}
$$

It follows that $\mathcal{D}^{-*} \supsetneq \mathcal{D}^{-}$since by allowing heterogeneity of sufficient subsets across individuals, we do not bar the former homogeneous configuration.

Let us also introduce the set of allocations in which no individual consumes all commodities:

$$
\overline{\bar{X}}:=\left\{x \in X \mid \forall i \in N, x_{i} \notin \mathbb{R}_{++}^{\ell}\right\}
$$

Notice that $\overline{\bar{X}} \supsetneq \bar{X}$. The restriction on allocations in $\overline{\bar{X}}$ seems very natural. Indeed, no ordinary individual would consume all commodities in the real world.

We then obtain the following variant of Theorem 1 .

Theorem 2 If a social ordering function satisfies Weak Pareto and Individual Independence of Irrelevant Commodities, then there is an agent who is a dictator for this social ordering function over $\left(\mathcal{D}^{-*}, X\right)$ and over $\left(\mathcal{R}^{n}, \overline{\bar{X}}\right)$.

The above theorem is also tight in the sense that $\mathcal{D}^{-*}$ is a maximal domain of dictatorship for $X$, and that $\overline{\bar{X}}$ is a maximal scope of dictatorship for $\mathcal{R}^{n}$. That is, there exist a social ordering function that is not dictatorial over $\left(\left\{R_{N}\right\}, X\right)$ for every $R_{N} \in \mathcal{D}^{+*}:=\mathcal{R}^{n} \backslash \mathcal{D}^{-*}$, and another social ordering function that is not dictatorial over $\left(\mathcal{R}^{n},\{x, y\}\right)$ for every pair $\{x, y\} \subseteq X \backslash \overline{\bar{X}}$ except those such that $x_{i}>y_{i}$ for all $i \in N$ or $x_{i}<y_{i}$ for all $i \in N$, and those such that $x_{i}=y_{i}$ for some $i \in N$.

IIIC may be criticized for restricting information about individual preferences too much. In fact, there are several different situations where individuals have zero units of a certain good: (i) people are simply not interested in the good and the good is not available in the local market, (ii) the good is available in the local market, but a particular individual does not want to consume it, or (iii) the good is normally available in the 
local market and everyone needs it, but it is in a serious shortage at a particular period (such as food during a period of famine). One may argue, for instance, that Japanese preferences over commodities that are normally unavailable in the Japanese market have to be ignored (case (i)), but if a particular individual in Japan does not consume sodas, which are available in the Japanese market, this tells something about her preferences about sodas, and it should be taken into account (case (ii)). Under IIIC, however, the individual's preferences for sodas are ignored in this context. The following subgroup (as opposed to individual) version of the independence condition would remedy this problem. Let $\left\{S_{1}, \ldots, S_{m}\right\}$ be a partition of the whole population $N$.

Subgroup Independence of Irrelevant Commodities: $\forall R_{N}, R_{N}^{\prime} \in \mathcal{R}^{n}, \forall x, y \in \mathbb{R}_{+}^{\ell n}$, if $\forall T \in\left\{S_{1}, \ldots, S_{m}\right\}, \exists K_{T} \subseteq L$ such that $\forall i \in T, x_{i}, y_{i} \in \mathbb{R}_{+}^{K_{T}}$ and $R_{i}$ and $R_{i}^{\prime}$ agree on $\mathbb{R}_{+}^{K_{T}}$, then $R$ and $R^{\prime}$ agree on $\{x, y\}$.

As already explained, we have adopted the individual version for simplicity. The subgroup version makes more room for possibility and less room for impossibility than in Theorem 2, and the larger each subgroup is, the more room we obtain for possibility. Formally, the theorem would hold on a domain between $\mathcal{D}^{-}$and $\mathcal{D}^{-*}$, and closer to $\mathcal{D}^{-}$ when independence is defined for larger subgroups, i.e. a coarser partition of $N$. A similar remark applies to the set $\overline{\bar{X}}$.

As for case (iii) above, one may argue that preferences for food during a period of famine should be taken into account when evaluating social desirability of allocations. Even IIC, however, requires that social rankings of allocations be independent of such preferences. We can take care of this problem with a slight weakening of the condition, allowing social orderings to depend not only on the available goods but also on those goods that are "familiar" to individuals. This extension of the informational basis of social ordering functions will be discussed in the next section in more details. 
Another remark should be made on the informational basis of social ordering functions under the independence condition. In the introduction, IIC has been contrasted with IIA by noticing that with IIC, but not with IIA, it is possible to refer to efficiency and fairness properties of allocations. On this account, IIIC occupies an intermediate position between IIA and IIC. To see this, consider world allocations in which the French and the Japanese consume different fixed subsets of commodities. The smaller the intersection of these subsets, the harder it is to assess efficiency and equity properties of such allocations. In the extreme case when the intersection is null, i.e. when they consume totally different commodities, it is then impossible to know, for instance, whether a French envies a Japanese on the sole basis of her preferences over French commodities.

\section{Anonymous social ordering functions}

In this final section, we explore the possibility of social ordering functions that are not only non-dictatorial, but also anonymous. The anonymity requirement on which we focus here is the mild condition that when two agents have the same preferences, it should be a matter of social indifference to permute their bundles, since, apart from their names, these agents are identical.

Anonymity: $\forall R_{N} \in \mathcal{R}^{n}, \forall x, y \in \mathbb{R}_{+}^{\ell n}, \forall i, j \in N$, if $R_{i}=R_{j}, y_{i}=x_{j}, y_{j}=x_{i}$ and $\forall k \neq i, j, y_{k}=x_{k}$, then $x I y$.

In order to obtain possibility results, social choice theory often looks at preference domain restrictions. But in the present case, there seems to be little hope for obtaining anonymous social ordering functions by only restricting the preference domain. In particular, the restriction to $\mathcal{D}^{+}$does not suffice. Consider a two-agent (Robinson, Friday) and $\ell$-commodity economy, which relates to the example in Section 2 . In $\mathcal{D}^{+}$and, presumably, 
in every reasonable domain $\mathcal{D}$, one can find two subsets $K, K^{\prime} \subseteq L$ such that $K \cap K^{\prime}=\emptyset$, and a profile $\left(R_{R}, R_{F}\right)$ such that $R_{R}$ and $R_{F}$ agree on $\mathbb{R}_{+}^{K}$ and on $\mathbb{R}_{+}^{K^{\prime}}$ separately but not on $\mathbb{R}_{+}^{K} \cup \mathbb{R}_{+}^{K^{\prime}}$, so that there are two pairs $q, q^{\prime} \in \mathbb{R}_{+}^{K}$ and $s, s^{\prime} \in \mathbb{R}_{+}^{K^{\prime}}$ ranked as follows: $q^{\prime} P_{R} s^{\prime} P_{R} s P_{R} q$ and $s^{\prime} P_{F} q^{\prime} P_{F} q P_{F} s$. Consider the following allocations:

\begin{tabular}{|c|c|c|}
\cline { 2 - 3 } \multicolumn{1}{c|}{} & Robinson & Friday \\
\hline$x$ & $q$ & $q^{\prime}$ \\
\hline$y$ & $q^{\prime}$ & $q$ \\
\hline$z$ & $s$ & $s^{\prime}$ \\
\hline$w$ & $s^{\prime}$ & $s$ \\
\hline
\end{tabular}

Suppose that there exists a social ordering function satisfying Weak Pareto, IIC and Anonymity. Then, by IIC and Anonymity, we have $x I y$ as well as $z I w$ since these pairs of allocation simply permute bundles for agents with identical preferences on the relevant subspace. But by Weak Pareto, z $P x$ and $y P w$, and together with $x I y$ and transitivity, we have $z P w$, which is a contradiction.

Whenever we find the above configuration somewhere in the space of allocations, we are unable to construct anonymous social preferences. The key fact underlying this example is that even though the two preference relations agree on each subspace, one is more sensitive than the other to differences in one subspace as compared to differences in the other subspace. It seems difficult to imagine a reasonable domain restriction that would preclude variations of sensitiveness across subspaces.

Preference domain restrictions, therefore, are not effective to obtain anonymous social ordering functions unless they are supplemented by other restrictions. We consider two kinds of additional restrictions here. The first is to restrict the application of the independence condition to certain commodities. The second is to restrict the set of allocations to be ranked by the social ordering function. We now show that combining 
a domain restriction with one or the other additional restriction provides two ways of obtaining a solution.

The key idea in these two strategies is to consider a "core" subset $K_{0}$ of commodities. Both restrict the domain to preferences for which this subset is sufficient:

$$
\mathcal{D}_{K_{0}}:=\left\{R_{N} \in \mathcal{R}^{n} \mid \forall i \in N, K_{0} \text { is sufficient for } R_{i}\right\}
$$

Then we define two social ordering functions, $\Psi_{K_{0}}^{*}$ and $\Psi_{K_{0}}^{* *}$, defined on $\mathcal{D}_{K_{0}}$ as follows. Take a vector $x_{0} \in \mathbb{R}_{++}^{K_{0}}$. Because $K_{0}$ is a sufficient subset of commodities, the index $v_{R_{i}}\left(\cdot ; x_{0}\right)$ introduced in Section 5 is always well defined. Like the Pazner-Schmeidler social ordering function, both $\Psi_{K_{0}}^{*}$ and $\Psi_{K_{0}}^{* *}$ rank allocations by the following rule: $x R y$ if and only if $\min _{i \in N} v_{R_{i}}\left(x_{i} ; x_{0}\right) \geq \min _{i \in N} v_{R_{i}}\left(y_{i} ; x_{0}\right){ }^{10}$

The difference between $\Psi_{K_{0}}^{*}$ and $\Psi_{K_{0}}^{* *}$ lies in the following. For all $R_{N} \in \mathcal{D}_{K_{0}}, \Psi_{K_{0}}^{*}\left(R_{N}\right)$ ranks all the allocations in $\mathbb{R}_{+}^{\ell n}$, whereas $\Psi_{K_{0}}^{* *}$ is defined so as to only rank the allocations belonging to the subset $X_{K_{0}}$ of allocations in which all commodities in $K_{0}$ are consumed in positive quantity:

$$
X_{K_{0}}:=\left\{x \in \mathbb{R}_{+}^{\ell n} \mid \forall k \in K_{0}, \sum_{i \in N} x_{i k}>0\right\} .
$$

The social ordering function $\Psi_{K_{0}}^{*}$ violates IIC and IIIC, but satisfies a weak version of these axioms, requiring that the social ranking of any two allocations should remain the same only when individual preferences over consumed commodities ${ }^{11}$ and the commodities in $K_{0}$ are unchanged.

The social ordering function $\Psi_{K_{0}}^{* *}$ satisfies IIC because commodities in $K_{0}$ are never absent in the allocations it ranks, so that the changes of preferences that are relevant

\footnotetext{
${ }^{10}$ In this way, these social ordering functions are not only anonymous but can also be considered "fair" in relation to the egalitarian-equivalent concept of fairness.

${ }^{11}$ That is, commodities consumed by society in the weak version of IIC, and consumed by the individual in the weak version of IIIC.
} 
to IIC never alter the preferences on the subspace $\mathbb{R}_{+}^{K_{0}}$, and hence the indices $v_{R_{i}}\left(\cdot ; x_{0}\right)$, either. By restricting further the set of allocations ranked by the social ordering function to those such that every agent consumes all commodities in $K_{0}$, one can obtain a third social ordering function satisfying IIIC.

Whether these solutions are applicable or not in any specific context depends on the possibility of finding an appropriate core subset $K_{0}$ of commodities. The subset $K_{0}$ must not only be rich enough to justify the preference domain restriction $\mathcal{D}_{K_{0}}$, but it must also be either "familiar" enough to justify the above weakening of the independence axioms, or "common" enough to justify the restriction of the set of allocations to be ranked by the social ordering function.

As in Section 5, let us briefly explore the implications of these results for model specification in social evaluation. Once again, perspectives are bleak with ordinary commodities. The diversity of commodities is simply overwhelming, so that it is hopeless to seek a core subset of ordinary commodities.

A better outlook appears when the objects of individual preferences are not ordinary commodities, but composite commodities. The more broadly the composite commodities are defined, the easier one can find a subset of composite commodities that are familiar or common as well as sufficient for all individuals. A drawback of composite commodities is that individual preferences over them are consistent only under severe restrictions. One must assume either that prices are fixed over all contemplated allocations, or that preferences are separable. Moreover, the more broadly the composite commodities are defined, the more severe the problem is.

At this point it is interesting to consider the alternative concepts of characteristics (Lancaster, 1971) or functionings (Sen, 1985). Both have the interesting feature that a given characteristic or functioning can be obtained with very different commodities. 
Moreover, these concepts are closely related to the physiology of human beings, which suffers less from variations across space and time than the list of commodities. As a consequence, it appears more promising, with characteristics or functionings, to find a sufficient "core" of dimensions for which either (i) preferences can always be estimated, justifying the restriction on the independence axioms, or (ii) consumption is always positive, justifying the restriction on the set of allocations to be ranked. As a simple example, consider the following (incomplete) list of functionings related to food: living without calorie deficiency, protein intake, entertaining others in a social meeting, etc. These basic functionings can be obtained at similar levels with very different types of food. They are present in positive quantities for all human beings in ordinary conditions, and even when they are absent, they are so familiar that individual preferences over them could always be estimated.

In general, the space of characteristics or functionings might be as diverse as the space of commodities. Indeed, Sen (1992) defines a functioning as any kind of achievement ("beings" and "doings") of a person. Therefore, we cannot claim that substituting functionings for commodities automatically eliminates the difficulties discussed in this paper. Nevertheless, focusing on basic or core characteristics or functionings, we can make some of the routes toward possibility easier to trod than with commodities. If that is true, a main conclusion emerging from the present analysis is that welfare economics would find better prospects for the construction of appealing criteria to evaluate social states if it migrated from the space of commodities to the space of characteristics or to the space of functionings. 


\section{References}

Arrow, K.J. (1950): A Difficulty in the Concept of Social Welfare, Journal of Political Economy, 58, 328-346.

Arrow, K.J. (1951): Social Choice and Individual Values. New York: Wiley. Second ed., 1963.

Border, K.C. (1983): Social Welfare Functions for Economic Environments With and Without the Pareto Principle, Journal of Economic Theory, 29, 205-216.

Bordes, G., and M. Le Breton (1989): Arrovian Theorems With Private Alternatives Domains and Selfish Individuals, Journal of Economic Theory, 47, 257-281.

Debreu, G. (1959): Theory of Value. New York: Wiley.

Donaldson, D., and J.E. Roemer (1987): Social Choice in Economic Environments With Dimensional Variation, Social Choice and Welfare, 4, 253-276.

Fleurbaey, M. (2003): Social Choice and Just Institutions: New Perspectives, forthcoming in Economics and Philosophy.

Fleurbaey, M. (2005): The Pazner-Schmeidler Social Ordering: A Defense, Review of Economic Design, 9, 145-166.

Fleurbaey, M., and F. Maniquet (2005): Fair Social Orderings with Unequal Production Skills, Social Choice and Welfare, 24, 1-35.

Fleurbaey, M., K. Suzumura and K. Tadenuma (2005a): Arrovian Aggregation in Economic Environments: How Much Should We Know About Indifference Surfaces?, Journal of Economic Theory, 124, 22-44.

Fleurbaey, M., K. Suzumura and K. Tadenuma (2005b): The Informational Basis of The Theory of Fair Allocation, Social Choice and Welfare, 24, 311-342.

Foley, D. (1967): Resource Allocation and the Public Sector, Yale Economic Essays, 7, 45-98. 
Kalai, E., E. Muller, and M.A. Satterthwaite (1979): Social Welfare Functions When Preferences Are Convex, Strictly Monotonic, and Continuous, Public Choice, 34, 87-97. Kolm, S.C. (1972): Justice et Equité. Paris: Ed. du CNRS.

Lancaster, K. (1971): Consumer Demand: A New Approach. New York: Columbia University Press.

Le Breton, M. (1997): Arrovian Social Choice on Economic Domains, in Social Choice Re-examined, Vol. 1, ed. by K.J. Arrow, A. Sen and K. Suzumura. London: Macmillan and New York: St. Martin's Press.

Le Breton, M., and J.A. Weymark (2000): Arrovian Social Choice Theory on Economic Domains, forthcoming in Handbook of Social Choice and Welfare, Vol. 2, ed. by K.J. Arrow, A. Sen and K. Suzumura. Amsterdam: North-Holland.

Mayston, D.J. (1974): The Idea of Social Choice. London: Macmillan.

Mayston, D.J. (1982): The Generation of a Social Welfare Function Under Ordinal Preferences, Mathematical Social Sciences, 3, 109-129.

Pazner, E. (1979): Equity, Nonfeasible Alternatives and Social Choice: A Reconsideration of the Concept of Social Welfare, in Aggregation and Revelation of Preferences, ed. by J.J. Laffont. Amsterdam: North-Holland.

Pazner, E., and D. Schmeidler (1978): Egalitarian-Equivalent Allocations: A New Concept of Economic Equity, Quarterly Journal of Economics, 92, 671-687.

Redekop, J. (1991): Social Welfare Functions on Restricted Economic Domains, Journal of Economic Theory, 53, 396-427.

Sen, A.K. (1970): Collective Choice and Social Welfare. San-Francisco: Holden-Day; republished Amsterdam: North-Holland, 1979.

Sen, A.K. (1985): Commodities and Capabilities. Amsterdam: North-Holland.

Sen, A.K. (1992): Inequality Reexamined. Oxford: Clarendon Press. 
Tadenuma, K. (2005): Egalitarian-Equivalence and the Pareto Principle for Social Preferences, Social Choice and Welfare, 24, 455-473.

\section{Appendix: Proofs}

Some additional notations are needed. For any $R_{i} \in \mathcal{R}$ and any $x_{i} \in \mathbb{R}_{+}^{\ell}$, the (closed) upper contour set for $R_{i}$ at $x_{i}$ is defined as

$$
u c\left(x_{i} ; R_{i}\right):=\left\{y_{i} \in \mathbb{R}_{+}^{\ell} \mid y_{i} R_{i} x_{i}\right\}
$$

For any $x_{i} \in \mathbb{R}_{+}^{\ell}$, the cone generated by $x_{i}$ is defined as

$$
C\left(x_{i}\right):=\left\{y_{i} \in \mathbb{R}_{+}^{\ell} \mid \exists \alpha \in \mathbb{R}_{+}, y_{i}=\alpha x_{i}\right\}
$$

In the proofs we sometimes consider social ordering functions defined on subdomains (i.e. proper subsets of $\mathcal{R}^{n}$ ). When we say that they satisfy an axiom, this means that they satisfy the axiom on their subdomain.

Proof of Theorem 1: We first prove separately dictatorship over $\left(\mathcal{D}^{-}, X\right)$ and over $\left(\mathcal{R}^{n}, \bar{X}\right)$. The proof relies on Arrow's theorem, and we need to define a variant of IIA. ${ }^{12}$

Weak Independence of Irrelevant Alternatives (WIIA): $\forall R_{N}, R_{N}^{\prime} \in \mathcal{R}^{n}, \forall x, y \in$ $X$, if $\forall i \in N, R_{i}$ and $R_{i}^{\prime}$ agree on $\{x, y\}$, and for no $i, x_{i} I_{i} y_{i}$, then $R$ and $R^{\prime}$ agree on $\{x, y\}$.

For any $K \subseteq L$, let $\mathcal{R}_{K} \subseteq \mathcal{R}$ denote the set of preference relations for which $K$ is sufficient.

\footnotetext{
${ }^{12}$ Our proof is influenced by Bordes and Le Breton (1989), who themselves adopt the "local approach" developed in Kalai et al. (1979). But we take advantage of a precise framework, whereas Bordes and Le Breton prove general results applying to several economic domains, and our much weaker independence condition requires new arguments.
} 
Lemma 1 Let $K \subsetneq L$ be given. On the domain $\left(\mathcal{R}_{K}\right)^{n}$, if a social ordering function satisfies Weak Pareto and Independence of Irrelevant Commodities, then it satisfies Weak Independence of Irrelevant Alternatives.

Proof of Lemma 1. Let $R_{N}, R_{N}^{\prime} \in\left(\mathcal{R}_{K}\right)^{n}$ and $x, y \in X$ be such that for all $i \in N, R_{i}$ and $R_{i}^{\prime}$ agree on $\{x, y\}$, and for no $i \in N, x_{i} I_{i} y_{i}$. Assume that $x P y$.

Let $M:=L \backslash K$. Since $x, y>0$, by strict monotonicity of preferences we know that $y_{i} P_{i} 0$ and $x_{i} P_{i}^{\prime} 0$ for all $i \in N$. Since $K$ is sufficient for all $i$, by strict monotonicity and continuity of preferences we can choose $z, w, z^{\prime}, w^{\prime} \in\left(\mathbb{R}_{+}^{K} \backslash\{0\}\right)^{n}$ such that for all $i \in N$, (i) if $x_{i} P_{i} y_{i}$ (and hence $x_{i} P_{i}^{\prime} y_{i}$ as well), then $z_{i} P_{i} x_{i} P_{i} y_{i} P_{i} w_{i}$ and $x_{i} P_{i}^{\prime} z_{i}^{\prime} P_{i}^{\prime} w_{i}^{\prime} P_{i}^{\prime} y_{i}$,

(ii) if $y_{i} P_{i} x_{i}$ (and hence $y_{i} P_{i}^{\prime} x_{i}$ as well), then $y_{i} P_{i} w_{i} P_{i} z_{i} P_{i} x_{i}$ and $w_{i}^{\prime} P_{i}^{\prime} y_{i} P_{i}^{\prime} x_{i} P_{i}^{\prime} z_{i}^{\prime}$, and

(iii) there are $\lambda_{i}, \lambda_{i}^{\prime} \in \mathbb{R}_{++}$such that $w_{i}=\lambda_{i} z_{i}, w_{i}^{\prime}=\lambda_{i}^{\prime} z_{i}^{\prime}$.

By Weak Pareto, we have $z P x$ and $y P w$. By transitivity of $P, z P w$. It also follows from Weak Pareto that $x P^{\prime} z^{\prime}$ and $w^{\prime} P^{\prime} y$.

Next, choose $a, b, a^{\prime}, b^{\prime}, a^{\prime \prime}, b^{\prime \prime} \in\left(\mathbb{R}_{+}^{M} \backslash\{0\}\right)^{n}$ such that for all $i \in N$,

(i) if $x_{i} P_{i} y_{i}$, then $a_{i}^{\prime}>a_{i}^{\prime \prime}>a_{i}>b_{i}>b_{i}^{\prime \prime}>b_{i}^{\prime}$, and

(ii) if $y_{i} P_{i} x_{i}$, then $b_{i}>b_{i}^{\prime \prime}>b_{i}^{\prime}>a_{i}^{\prime}>a_{i}^{\prime \prime}>a_{i}$. and

(iii) there are $\mu_{i}, \mu_{i}^{\prime} \in \mathbb{R}_{++}$such that $b_{i}=\mu_{i} a_{i}, b_{i}^{\prime}=\mu_{i}^{\prime} a_{i}^{\prime}$.

For every $i \in N$, choose two increasing functions $\gamma_{i}, \gamma_{i}^{\prime}: \mathbb{R}_{+} \rightarrow \mathbb{R}_{+}$such that $\gamma_{i}(0)=$ $\gamma_{i}^{\prime}(0)=0, \gamma_{i}(1)=\gamma_{i}^{\prime}(1)=1$ and $\gamma_{i}\left(\lambda_{i}\right)=\mu_{i}, \gamma_{i}^{\prime}\left(\lambda_{i}^{\prime}\right)=\mu_{i}^{\prime}$. Such functions always exist because $\lambda_{i}>1 \Leftrightarrow \mu_{i}>1$ and $\lambda_{i}^{\prime}>1 \Leftrightarrow \mu_{i}^{\prime}>1$.

Let $R_{0} \in \mathcal{R}$ be an arbitrary preference relation on $\mathbb{R}_{+}^{\ell}$. We now define a new preference relation for $i, R_{i}^{*}$, as follows. The upper contour set for $R_{i}^{*}$ at any $q \in C\left(z_{i}\right)$ such that $q=\alpha z_{i}$ for some $\alpha$ is constructed as

$$
u c\left(q ; R_{i}^{*}\right):=c o\left\{\left(u c\left(q ; R_{i}\right) \cap \mathbb{R}_{+}^{K}\right) \cup\left(u c\left(\gamma_{i}(\alpha) a_{i} ; R_{0}\right) \cap \mathbb{R}_{+}^{M}\right)\right\}
$$


where co denotes the convex hull. More generally, for any $c \in \mathbb{R}_{+}^{\ell}$, we define

$$
u c\left(c ; R_{i}^{*}\right):=\bigcap_{q \in C\left(z_{i}\right), c \in u c\left(q ; R_{i}^{*}\right)} u c\left(q ; R_{i}^{*}\right) .
$$

As a convex hull, $u c\left(q ; R_{i}^{*}\right)$ is convex for all $q \in C\left(z_{i}\right)$, and as an intersection of convex sets, $u c\left(c ; R_{i}^{*}\right)$ is convex for all $c \in \mathbb{R}_{+}^{\ell}$. This means that $R_{i}^{*}$ is convex. Clearly, it is also continuous and strictly monotonic, so that $R_{i}^{*} \in \mathcal{R}$. Moreover, $R_{i}^{*}$ agrees with $R_{i}$ on $\mathbb{R}_{+}^{K}$. Indeed, if $c \in \mathbb{R}_{+}^{K}$,

$$
\begin{aligned}
u c\left(c ; R_{i}^{*}\right) \cap \mathbb{R}_{+}^{K} & =\bigcap_{q \in C\left(z_{i}\right), c \in u c\left(q ; R_{i}^{*}\right)} u c\left(q ; R_{i}^{*}\right) \cap \mathbb{R}_{+}^{K} \\
& =\bigcap_{q \in C\left(z_{i}\right), c \in u c\left(q ; R_{i}\right) \cap \mathbb{R}_{+}^{K}} u c\left(q ; R_{i}\right) \cap \mathbb{R}_{+}^{K} \\
& =u c\left(c ; R_{i}\right) \cap \mathbb{R}_{+}^{K} .
\end{aligned}
$$

Similarly, $R_{i}^{*}$ agrees with $R_{0}$ on $\mathbb{R}_{+}^{M}$. Finally, $z_{i} I_{i}^{*} a_{i}$, because

$$
\begin{aligned}
u c\left(z_{i} ; R_{i}^{*}\right) \cap \mathbb{R}_{+}^{M} & =c o\left\{\left(u c\left(z_{i} ; R_{i}\right) \cap \mathbb{R}_{+}^{K}\right) \cup\left(u c\left(\gamma_{i}(1) a_{i} ; R_{0}\right) \cap \mathbb{R}_{+}^{M}\right)\right\} \cap \mathbb{R}_{+}^{M} \\
& =c o\left\{\left(u c\left(z_{i} ; R_{i}\right) \cap \mathbb{R}_{+}^{K}\right) \cup\left(u c\left(a_{i} ; R_{0}\right) \cap \mathbb{R}_{+}^{M}\right)\right\} \cap \mathbb{R}_{+}^{M} \\
& =u c\left(a_{i} ; R_{0}\right) \cap \mathbb{R}_{+}^{M},
\end{aligned}
$$

and $w_{i} I_{i}^{*} b_{i}$, because

$$
\begin{aligned}
u c\left(w_{i} ; R_{i}^{*}\right) \cap \mathbb{R}_{+}^{M} & =c o\left\{\left(u c\left(w_{i} ; R_{i}\right) \cap \mathbb{R}_{+}^{K}\right) \cup\left(u c\left(\gamma_{i}\left(\lambda_{i}\right) a_{i} ; R_{0}\right) \cap \mathbb{R}_{+}^{M}\right)\right\} \cap \mathbb{R}_{+}^{M} \\
& =c o\left\{\left(u c\left(w_{i} ; R_{i}\right) \cap \mathbb{R}_{+}^{K}\right) \cup\left(u c\left(b_{i} ; R_{0}\right) \cap \mathbb{R}_{+}^{M}\right)\right\} \cap \mathbb{R}_{+}^{M} \\
& =u c\left(b_{i} ; R_{0}\right) \cap \mathbb{R}_{+}^{M}
\end{aligned}
$$

Similarly, we construct $R_{i}^{* *} \in \mathcal{R}$ by

$$
u c\left(q ; R_{i}^{\prime *}\right):=c o\left\{\left(u c\left(q ; R_{i}^{\prime}\right) \cap \mathbb{R}_{+}^{K}\right) \cup\left(u c\left(\gamma_{i}^{\prime}(\alpha) a_{i}^{\prime} ; R_{0}\right) \cap \mathbb{R}_{+}^{M}\right)\right\}
$$

for $q \in C\left(z_{i}^{\prime}\right), q=\alpha z_{i}^{\prime}$, and

$$
u c\left(c ; R_{i}^{* *}\right):=\bigcap_{q \in C\left(z_{i}^{\prime}\right), c \in u c\left(q ; R_{i}^{\prime *}\right)} u c\left(q ; R_{i}^{\prime *}\right) .
$$


for any $c \in \mathbb{R}_{+}^{\ell}$. The ordering $R_{i}^{\prime *}$ agrees with $R_{i}^{\prime}$ on $\mathbb{R}_{+}^{K}$, and with $R_{0}$ on $\mathbb{R}_{+}^{M}$. Moreover, $z_{i}^{\prime} I_{i}^{* *} a_{i}^{\prime}$ and $w_{i}^{\prime} I_{i}^{\prime *} b_{i}^{\prime}$. Since both $R_{i}^{*}$ and $R_{i}^{\prime *}$ agree with $R_{0}$ on $\mathbb{R}_{+}^{M}$, they agree with each other on $\mathbb{R}_{+}^{M}$ as well. Let $R_{N}^{*}:=\left(R_{1}^{*}, \ldots, R_{n}^{*}\right)$ and $R_{N}^{\prime *}:=\left(R_{1}^{\prime *}, \ldots, R_{n}^{* *}\right)$. By construction, $R_{N}^{*}, R_{N}^{*} \in\left(\mathcal{R}_{K}\right)^{n}$

Recall that $a_{i}^{\prime \prime}>a_{i}$ and $b_{i}>b_{i}^{\prime \prime}$. By transitivity and strict monotonicity of preferences, $a_{i}^{\prime \prime} P_{i}^{*} z_{i}$ and $w_{i} P_{i}^{*} b_{i}^{\prime \prime}$ for all $i \in N$. By Weak Pareto, $a^{\prime \prime} P^{*} z$ and $w P^{*} b^{\prime \prime}$. Since $z P w$, it follows from Independence of Irrelevant Commodities (IIC) that $z P^{*} w$. By transitivity of $R^{*}$, we have $a^{\prime \prime} P^{*} b^{\prime \prime}$.

Next recall that $a_{i}^{\prime}>a_{i}^{\prime \prime}$ and $b_{i}^{\prime \prime}>b_{i}^{\prime}$. By transitivity and strict monotonicity of preferences and Weak Pareto, $z^{\prime} P_{i}^{\prime *} a^{\prime \prime}$ and $b^{\prime \prime} P_{i}^{\prime *} w^{\prime}$. On the other hand, it follows from IIC and $a^{\prime \prime} P^{*} b^{\prime \prime}$ that $a^{\prime \prime} P^{*} b^{\prime \prime}$. By transitivity of $P^{\prime *}, z^{\prime} P^{* *} w^{\prime}$. From IIC (applied to $R_{N}^{*}$ and $R_{N}^{\prime}$ ), we have $z^{\prime} P^{\prime} w^{\prime}$. Recall that $x P^{\prime} z^{\prime}$ and $w^{\prime} P^{\prime} y$. By transitivity, $x P^{\prime} y$.

We have shown that $x P y \Rightarrow x P^{\prime} y$. By symmetry, $x P^{\prime} y \Rightarrow x P y$, and $y P x \Leftrightarrow$ $y P^{\prime} x$. Hence, it also holds that $x I y \Leftrightarrow x I^{\prime} y$.

Let $\mathcal{D} \subseteq \mathcal{R}^{n}$ and $Y \subseteq X$ be given. An agent $i_{0} \in N$ is called a quasi-dictator $\operatorname{over}(\mathcal{D}, Y)$ if for all $R_{N} \in \mathcal{D}$, and all $x, y \in Y, x P y$ whenever $x_{i_{0}} P_{i_{0}} y_{i_{0}}$ and for no $i \in N, x_{i} I_{i} y_{i}{ }^{13}$ In the sequel, when $\mathcal{D}$ is not specified for a quasi-dictator or a dictator, this means that the domain of the social ordering function is the relevant set. A pair of allocations $\{x, y\} \subseteq X$ is called a trivial pair on $\mathcal{D}$ if there is $i \in N$ such that for all $R_{N}, R_{N}^{\prime} \in \mathcal{D}, R_{i}$ and $R_{i}^{\prime}$ agree on $\{x, y\}$. By strict monotonicity of preferences, this happens when either $x>y$ or $x<y$. A set of three allocations $\{x, y, z\} \subseteq X$ is called a free triple on $\mathcal{D}$ if for every $n$-tuple of orderings $O_{N}$ on $\{x, y, z\}$, there exists $R_{N} \in \mathcal{D}$ such that $R_{N}$ and $O_{N}$ agree on $\{x, y, z\}$.

\footnotetext{
${ }^{13}$ This was called a "strict dictator" in Redekop (1991), where a similar strategy of proof relying on linear orders was introduced.
} 
Lemma 2 Let $K \subseteq L$ be given. On the domain $\left(\mathcal{R}_{K}\right)^{n}$, if a social ordering function satisfies Weak Pareto and Weak Independence of Irrelevant Alternatives, then for every free triple $\{x, y, z\} \subseteq X$, there exists a quasi-dictator over $\{x, y, z\}$.

Proof of Lemma 2. Consider a social ordering function defined on $\left(\mathcal{R}_{K}\right)^{n}$ that satisfies Weak Pareto and WIIA. Let $\{x, y, z\} \subseteq X$ be a free triple on $\left(\mathcal{R}_{K}\right)^{n}$ and let $\left(\mathcal{L}_{\{x, y, z\}}\right)^{n}$ be the set of all profiles of linear orders (i.e., complete orderings without indifference) over $\{x, y, z\}$.

Consider two profiles $R_{N}, R_{N}^{\prime} \in\left(\mathcal{R}_{K}\right)^{n}$ that generate the same linear orders over $\{x, y, z\}$, i.e. for all $a, b \in\{x, y, z\}$ such that $a \neq b$, for all $i \in N$ one has

$$
a P_{i} b \Leftrightarrow a P_{i}^{\prime} b
$$

By WIIA, the social ordering function yields the same ranking of $\{x, y, z\}$ for $R_{N}$ and $R_{N}^{\prime}$. This means that this social ordering function induces a well-defined social ordering function on $\left(\mathcal{L}_{\{x, y, z\}}\right)^{n}$. The induced social ordering function satisfies Weak Pareto and IIA on this domain. By application of the variant of Arrow's theorem for linear orders, the induced social ordering function has a dictator $i_{0}$ over $\{x, y, z\}$.

This implies that $i_{0}$ is a quasi-dictator over $\{x, y, z\}$ for the initial social ordering function defined on $\left(\mathcal{R}_{K}\right)^{n}$.

Lemma 3 Let $K \subseteq L$ be given. Let $\{x, y\},\{z, w\} \subseteq X$ be non-trivial pairs on $\left(\mathcal{R}_{K}\right)^{n}$. There exist $v^{1}, \ldots, v^{m} \in X$ such that

$$
v^{1}=x, v^{2}=y, v^{m-1}=z, v^{m}=w
$$

and for all $q=1, \ldots, m-2,\left\{v^{q}, v^{q+1}, v^{q+2}\right\}$ is a free triple on $\left(\mathcal{R}_{K}\right)^{n}$.

Proof of Lemma 3. Since $\{x, y\}$ is a non-trivial pair, there exists $p \in \mathbb{R}_{++}^{\ell}$ such that 
$p x=p y$. Let

$$
\begin{aligned}
x^{\prime} & =\frac{2}{3} x+\frac{1}{3} y \\
y^{\prime} & =\frac{1}{3} x+\frac{2}{3} y .
\end{aligned}
$$

For every $\varepsilon \in \mathbb{R}_{++}$there exist $x^{\prime \prime}, y^{\prime \prime} \in\left(\mathbb{R}_{++}^{\ell}\right)^{n}$ such that $\left\|x^{\prime \prime}-x^{\prime}\right\|<\varepsilon,\left\|y^{\prime \prime}-y^{\prime}\right\|<\varepsilon$ (where $\|\cdot\|$ denotes the Euclidean norm), and $\{x, y, u\},\left\{v, x^{\prime \prime}, y^{\prime \prime}\right\}$ are free triples for every $u \in\left\{x^{\prime \prime}, y^{\prime \prime}\right\}$ and $v \in\{x, y\}$. Similarly, one constructs $z^{\prime \prime}, w^{\prime \prime} \in\left(\mathbb{R}_{++}^{\ell}\right)^{n}$ such that $\{z, w, u\},\left\{v, z^{\prime \prime}, w^{\prime \prime}\right\}$ are free triples for every $u \in\left\{z^{\prime \prime}, w^{\prime \prime}\right\}$ and $v \in\{z, w\}$.

The pairs $\left\{x^{\prime \prime}, y^{\prime \prime}\right\},\left\{z^{\prime \prime}, w^{\prime \prime}\right\}$ are non-trivial, with $x^{\prime \prime}, y^{\prime \prime}, z^{\prime \prime}, w^{\prime \prime} \in\left(\mathbb{R}_{++}^{\ell}\right)^{n}$. Pick $i \in N$. Let $\bar{p}, \bar{p}^{\prime} \in \mathbb{R}_{++}^{\ell}$ be such that $\bar{p} x_{i}^{\prime \prime}=\bar{p} y_{i}^{\prime \prime}$ and $\bar{p}^{\prime} z_{i}^{\prime \prime}=\bar{p}^{\prime} w_{i}^{\prime \prime}$. Consider the set

$$
B_{i}=\left\{q \in \mathbb{R}_{++}^{\ell} \mid \bar{p} q>\bar{p} x_{i}^{\prime \prime}, \bar{p}^{\prime} q>\bar{p}^{\prime} z_{i}^{\prime \prime}, q \ngtr x_{i}^{\prime \prime}, y_{i}^{\prime \prime}, z_{i}^{\prime \prime}, w_{i}^{\prime \prime}\right\} .
$$

Because $x_{i}^{\prime \prime}, y_{i}^{\prime \prime}, z_{i}^{\prime \prime}, w_{i}^{\prime \prime} \gg 0$, there exist $\bar{p}^{\prime \prime} \in \mathbb{R}_{++}^{\ell}$ and $q_{i}^{\prime}, q_{i}^{\prime \prime} \in B_{i}$ such that

$$
\bar{p}^{\prime \prime} q^{\prime}=\bar{p}^{\prime \prime} q^{\prime \prime}<\bar{p}^{\prime \prime} x_{i}^{\prime \prime}, \bar{p}^{\prime \prime} y_{i}^{\prime \prime}, \bar{p}^{\prime \prime} z_{i}^{\prime \prime}, \bar{p}^{\prime \prime} w_{i}^{\prime \prime}
$$

This construction can be made for every $i \in N$. One can check that $\left\{x^{\prime \prime}, y^{\prime \prime}, u\right\}$, $\left\{z^{\prime \prime}, w^{\prime \prime}, u\right\}$ and $\left\{v, q^{\prime}, q^{\prime \prime}\right\}$ are free triples for every $u \in\left\{q^{\prime}, q^{\prime \prime}\right\}$ and $v \in\left\{x^{\prime \prime}, y^{\prime \prime}, z^{\prime \prime}, w^{\prime \prime}\right\}$.

One can now connect $\{x, y\},\{z, w\}$ by the following sequence of free triples: $\left\{x, y, x^{\prime \prime}\right\},\left\{y, x^{\prime \prime}, y^{\prime \prime}\right\},\left\{x^{\prime \prime}, y^{\prime \prime}, q^{\prime}\right\},\left\{y^{\prime \prime}, q^{\prime}, q^{\prime \prime}\right\},\left\{q^{\prime}, q^{\prime \prime}, z^{\prime \prime}\right\},\left\{q^{\prime \prime}, z^{\prime \prime}, w^{\prime \prime}\right\}, \quad\left\{z^{\prime \prime}, w^{\prime \prime}, z\right\}$, $\left\{w^{\prime \prime}, z, w\right\}$.

Lemma 4 Let $K \subseteq L$ be given. Let $R_{N} \in\left(\mathcal{R}_{K}\right)^{n}$ and $x, y \in X$ be such that for no $i \in N$, $x_{i} I_{i} y_{i}$. Then, there exists $z \in X$ such that $\{x, z\}$ and $\{z, y\}$ are non-trivial on $\left(\mathcal{R}_{K}\right)^{n}$ and for all $i \in N$, either $x_{i} P_{i} z_{i} P_{i} y_{i}$ or $y_{i} P_{i} z_{i} P_{i} x_{i}$.

Proof of Lemma 4. Pick $i \in N$ and assume, without loss of generality, that $x_{i} P_{i} y_{i}$.

First case: $x_{i}>y_{i}$. Suppose, again without loss of generality, that $x_{i 1}>y_{i 1}$. 
First subcase: $y_{i 1}>0$. One can find $\varepsilon, \eta \in \mathbb{R}_{++}$such that

$$
z_{i}=\left(y_{i 1}-\varepsilon, x_{i 2}+\eta, y_{i 3}, \ldots, y_{i \ell}\right)
$$

satisfies $x_{i} P_{i} z_{i} P_{i} y_{i}$.

Second subcase: $y_{i 1}=0$ and (without loss of generality) $y_{i 2}>0$. One can find $\varepsilon, \eta \in \mathbb{R}_{++}$such that

$$
z_{i}=\left(x_{i 1}+\varepsilon, y_{i 2}-\eta, y_{i 3}, \ldots, y_{i \ell}\right)
$$

satisfies $x_{i} P_{i} z_{i} P_{i} y_{i}$.

Second case: $x_{i} \ngtr y_{i}$. For $\lambda \in(0,1)$, let

$$
z_{i}=\lambda x_{i}+(1-\lambda) y_{i}
$$

By convexity of preferences, for $\lambda$ close enough to 0 , one has $x_{i} P_{i} z_{i} P_{i} y_{i}$.

Lemma 5 Let $K \subseteq L$ be given. On the domain $\left(\mathcal{R}_{K}\right)^{n}$, suppose that for every free triple $\{x, y, z\} \subseteq X$, there is a quasi-dictator over $\{x, y, z\}$. Then there is a quasi-dictator over $X$.

Proof of Lemma 5. Pick any triple $\{a, b, c\} \subseteq X$ that is free on $\left(\mathcal{R}_{K}\right)^{n}$ and let $i_{0}$ be its quasi-dictator on $\left(\mathcal{R}_{K}\right)^{n}$. Let $R_{N} \in\left(\mathcal{R}_{K}\right)^{n}$ and $x, y \in X$ be such that $x_{i_{0}} P_{i_{0}} y_{i_{0}}$ and for no $i \in N, x_{i} I_{i} y_{i}$. By Lemma 4 , there is $z \in X$ such that $\{x, z\}$ and $\{z, y\}$ are non-trivial on $\left(\mathcal{R}_{K}\right)^{n}$ and for all $i \in N$, either $x_{i} P_{i} z_{i} P_{i} y_{i}$ or $y_{i} P_{i} z_{i} P_{i} x_{i}$. In particular, one has $x_{i_{0}} P_{i_{0}} z_{i_{0}} P_{i_{0}} y_{i_{0}}$.

By Lemma 3, there exist $v^{1}, \ldots, v^{m} \in X$ such that

$$
v^{1}=a, v^{2}=b, v^{m-1}=x, v^{m}=z
$$

and for all $q=1, \ldots, m-2,\left\{v^{q}, v^{q+1}, v^{q+2}\right\}$ is a free triple on $\left(\mathcal{R}_{K}\right)^{n}$. Similarly, there exist $w^{1}, \ldots, w^{t} \in X$ such that

$$
w^{1}=a, w^{2}=b, w^{t-1}=y, w^{t}=z
$$


and for all $q=1, \ldots, t-2,\left\{w^{q}, w^{q+1}, w^{q+2}\right\}$ is a free triple on $\left(\mathcal{R}_{K}\right)^{n}$.

Necessarily $i_{0}$ is a quasi-dictator for all $\left\{v^{q}, v^{q+1}, v^{q+2}\right\}$, for $q=1, \ldots, m-2$, as well as for all $\left\{w^{q}, w^{q+1}, w^{q+2}\right\}$, for $q=1, \ldots, t-2$. This implies that $i_{0}$ is a quasi-dictator over $\{x, z\}$ and over $\{z, y\}$. Therefore, $x P z$ and $z P y$. By transitivity, $x P y$.

Lemma 6 Let $K \subseteq L$ be given. On the domain $\left(\mathcal{R}_{K}\right)^{n}$, if $i_{0} \in N$ is a quasi-dictator over $X$, then $i_{0}$ is a dictator over $X$.

Proof of Lemma 6. Let $x, y \in X$ and $R_{N} \in\left(\mathcal{R}_{K}\right)^{n}$ be such that $x_{i_{0}} P_{i_{0}} y_{i_{0}}$. By continuity and strict monotonicity of preferences, there exists $z \in X$ such that $x_{i_{0}} P_{i_{0}} z_{i_{0}} P_{i_{0}} y_{i_{0}}$ and for all $i \in N$, either $x_{i} P_{i} z_{i} P_{i} y_{i}$ or $y_{i} R_{i} x_{i} P_{i} z_{i}$. It follows that $x P z$ (by Weak Pareto) and $z P y$ (because $i_{0}$ is a quasi-dictator). By transitivity, $x P y$.

We can now complete the proof of dictatorship over $\left(\mathcal{D}^{-}, X\right)$. Note that $\mathcal{D}^{-}=$ $\bigcup_{K \subsetneq L}\left(\mathcal{R}_{K}\right)^{n}$. Consider a social ordering function $\Psi$ defined on $\mathcal{R}^{n}$ that satisfies Weak Pareto and IIC. For every $K \subsetneq L$, its restriction $\Psi_{K}$ to the subdomain $\left(\mathcal{R}_{K}\right)^{n}$ also satisfies these conditions. It follows from Lemmas $1,2,5$ and 6 that for every $K \subsetneq L, \Psi_{K}$ has a dictator $i_{K}$ over $X$. This implies that for $\Psi$, for every $K \subsetneq L$ there is a dictator $i_{K}$ over $\left(\left(\mathcal{R}_{K}\right)^{n}, X\right)$. Suppose that there are $K, K^{\prime} \subsetneq L$ such that $i_{K} \neq i_{K^{\prime}}$. Let $x, y \in X$ and $R_{N} \in \bigcap_{K \subsetneq L, K \neq \emptyset}\left(\mathcal{R}_{K}\right)^{n}$ be such that $x_{i_{K}} P_{i_{K}} y_{i_{K}}$ and $y_{i_{K^{\prime}}} P_{i_{K^{\prime}}} x_{i_{K^{\prime}}}$. (Such a configuration is easy to obtain since any profile of linear preferences with positive normals belongs to $\bigcap_{K \subsetneq L, K \neq \emptyset}\left(\mathcal{R}_{K}\right)^{n}$.) One must have $x P y$ and $y P x$, which is impossible. Therefore the same agent must be the dictator for all $K \subsetneq L$, i.e. on $\mathcal{D}^{-}$. This proves that there is a dictator over $\left(\mathcal{D}^{-}, X\right)$.

Remark. This impossibility result no longer holds if the set $\mathcal{R}$ is extended to include preference relations that are monotonic but not strictly. Let $A\left(R_{N}\right)=$ $\left\{x \in X \mid \forall i \in N, x_{i} P_{i} 0\right\}$ and $B\left(R_{N}\right)=\left\{x \in X \mid \exists i \in N, x_{i} I_{i} 0\right\}$. Consider the following social ordering function: for all $x, y \in \mathbb{R}_{+}^{\ell n}, x R y$ whenever one of the conditions 
below holds:

(i) $x \in A\left(R_{N}\right)$ and $y \in B\left(R_{N}\right)$;

(ii) $x, y \in A\left(R_{N}\right)$ and $x_{1} R_{1} y_{1}$;

(iii) $x, y \in B\left(R_{N}\right)$ and $x_{2} R_{2} y_{2}$.

This social ordering function satisfies Weak Pareto and IIC, but is not dictatorial. However, this example obviously displays clear dictatorial features, and the essence of our results does not really depend on strict monotonicity of preferences. In particular on the subdomain of $\mathcal{D}^{-}$(extended to cover preferences that are not strictly monotonic) such that for every $i \in N$, and every $x_{i}>0, x_{i} P_{i} 0$, the dictatorship result is preserved.

Remark. The proof involves only profiles from $\mathcal{D}^{-}$. Therefore, it also shows that a social ordering function defined on the subdomain $\mathcal{D}^{-}$and satisfying Weak Pareto and IIC has a dictator over $X$.

The proof of dictatorship over $\left(\mathcal{R}^{n}, \bar{X}\right)$ relies on the following lemmas.

Lemma 7 Let $K \subsetneq L, K \neq \emptyset$ be given. On the domain $\mathcal{R}^{n}$, if a social ordering function satisfies Weak Pareto and Independence of Irrelevant Commodities, then it satisfies Weak Independence of Irrelevant Alternatives restricted to allocations in $\left(\mathbb{R}_{+}^{K} \backslash\{0\}\right)^{n}$.

Proof of Lemma 7: Let $x, y \in\left(\mathbb{R}_{+}^{K} \backslash\{0\}\right)^{n}$ and $R_{N}, R_{N}^{\prime} \in \mathcal{R}^{n}$ be such that for all $i \in N, R_{i}$ and $R_{i}^{\prime}$ agree on $\{x, y\}$ and for no $i \in N, x_{i} I_{i} y_{i}$. Let $M:=L \backslash K$. Suppose $x P y$.

By a similar method as in the proof of Lemma 1 , one constructs $R_{N}^{*}, R_{N}^{*} \in \mathcal{R}^{n}$ and $a, a^{\prime}, a^{\prime \prime}, b, b^{\prime}, b^{\prime \prime} \in \mathbb{R}_{+}^{M} \backslash\{0\}$ such that for all $i \in N$ :

(i) if $x_{i} P_{i} y_{i}$ then $a_{i}^{\prime}>a_{i}^{\prime \prime}>a_{i}>b_{i}>b_{i}^{\prime \prime}>b_{i}^{\prime}$;

(ii) if $y_{i} P_{i} x_{i}$ then $b_{i}>b_{i}^{\prime \prime}>b_{i}^{\prime}>a_{i}^{\prime}>a_{i}^{\prime \prime}>a_{i}$;

(iii) $R_{i}$ and $R_{i}^{*}$ agree on $\mathbb{R}_{+}^{K}$; 
(iv) $R_{i}^{\prime}$ and $R_{i}^{\prime *}$ agree on $\mathbb{R}_{+}^{K}$;

(v) $R_{i}^{*}$ and $R_{i}^{\prime *}$ agree on $\mathbb{R}_{+}^{M}$;

(vi) $x_{i} I_{i}^{*} a_{i}$ and $y_{i} I_{i}^{*} b_{i}$;

(vii) $x_{i} I_{i}^{\prime *} a_{i}^{\prime}$ and $y_{i} I_{i}^{\prime *} b_{i}^{\prime}$.

By Weak Pareto, $a^{\prime \prime} P^{*} x$ and $y P^{*} b^{\prime \prime}$. By IIC, $x P^{*} y$ so that $a^{\prime \prime} P^{*} b^{\prime \prime}$. By IIC again, $a^{\prime \prime} P^{\prime *} b^{\prime \prime}$. By Weak Pareto, $x P^{\prime *} a^{\prime \prime}$ and $b^{\prime \prime} P^{\prime *} y$ so that $x P^{\prime *} y$. By IIC, $x P^{\prime} y$. As in Lemma 1 , one then easily deduces that $R$ and $R^{\prime}$ agree on $\{x, y\}$.

Lemma 8 Let $K \subsetneq L$, with cardinality $|K|>1$. On the domain $\mathcal{R}^{n}$, if a social ordering function satisfies Weak Pareto and Weak Independence of Irrelevant Alternatives restricted to allocations in $\left(\mathbb{R}_{+}^{K} \backslash\{0\}\right)^{n}$, then there is a dictator over $\left(\mathbb{R}_{+}^{K} \backslash\{0\}\right)^{n}$.

This lemma can be proved as in the sequence of Lemmas 2, 5 and 6 .

Lemma 9 Let $K \subsetneq L,|K|=1$. On the domain $\mathcal{R}^{n}$, if a social ordering function satisfies Weak Pareto and Independence of Irrelevant Commodities, then there is a dictator over $\left(\mathbb{R}_{+}^{K} \backslash\{0\}\right)^{n}$

Proof of Lemma 9: By IIC and monotonicity of preferences, the social ranking over $\left(\mathbb{R}_{+}^{K} \backslash\{0\}\right)^{n}$ does not depend on individual preferences. Consider $x, y, z, w \in\left(\mathbb{R}_{+}^{K} \backslash\{0\}\right)^{n}$ such that for every $i \in N, x_{i} \gtrless y_{i}$ if and only if $z_{i} \gtrless w_{i}$ and for no $i \in N, x_{i}=y_{i}$.

Let $K^{\prime} \subseteq L \backslash K,\left|K^{\prime}\right|=1$. Let $a, b \in\left(\mathbb{R}_{+}^{K^{\prime}} \backslash\{0\}\right)^{n}$ be such that for every $i \in N$, $x_{i} \gtrless y_{i}$ if and only if $a_{i} \gtrless b_{i}$. Suppose $x P y$. One can construct $R_{N} \in \mathcal{R}^{n}$ such that for every $i \in N$, either $a_{i} P_{i} x_{i} P_{i} y_{i} P_{i} b_{i}$ or $y_{i} P_{i} b_{i} P_{i} a_{i} P_{i} x_{i}$. By Weak Pareto, $a P x$ and y $P b$, so that by transitivity, $a P b$. By IIC and monotonicity of preferences, this ranking does not depend on individual preferences since $a, b \in\left(\mathbb{R}_{+}^{K^{\prime}} \backslash\{0\}\right)^{n}$ and $\left|K^{\prime}\right|=1$. By a similar reasoning, one can show that $a P b$ implies $z P w$. Similarly, if $y P x$, one proves 
that $w P z$. In summary, $x P y$ if and only if $z P w$, and $y R x$ if and only if $w R z$. Let us call this property "neutrality".

The rest of the proof mimics part of the proof of Arrow's theorem (see, e.g., Sen 1970, ch. $\left.3^{*}\right)$. We present it for completeness. Take any $G \subseteq N$ such that for all $x, y \in\left(\mathbb{R}_{+}^{K} \backslash\{0\}\right)^{n}, x P y$ if $x_{i}>y_{i}$ for all $i \in G$. (This property holds for $G=N$ by Weak Pareto.) Partition $G$ into non-empty subsets $G_{1}$ and $G_{2}$. Let $x, y \in\left(\mathbb{R}_{+}^{K} \backslash\{0\}\right)^{n}$ be such that $x_{i}>y_{i}$ for all $i \in N \backslash G_{2}$ and $x_{i}<y_{i}$ for all $i \in G_{2}$. Construct $z \in\left(\mathbb{R}_{+}^{K} \backslash\{0\}\right)^{n}$ such that $x_{i}>y_{i}>z_{i}$ for all $i \in G_{1}, x_{i}<z_{i}<y_{i}$ for all $i \in G_{2}$ and $z_{i}>x_{i}>y_{i}$ for all $i \in N \backslash G$. One has $y P z$ because $y_{i}>z_{i}$ for all $i \in G$. Now, either $x P z$ or $z R x$. In the former case, by neutrality this implies that for all $a, b \in\left(\mathbb{R}_{+}^{K} \backslash\{0\}\right)^{n}, a P b$ whenever $a_{i}>b_{i}$ for all $i \in G_{1}$ and $a_{i}<b_{i}$ for all $i \in N \backslash G_{1}$. In the latter, this implies y $P x$, so that by neutrality, for all $a, b \in\left(\mathbb{R}_{+}^{K} \backslash\{0\}\right)^{n}, a P b$ whenever $a_{i}>b_{i}$ for all $i \in G_{2}$ and $a_{i}<b_{i}$ for all $i \in N \backslash G_{2}$.

Let us pursue the former case. (A similar argument applies to the other case.) Let $a, b \in\left(\mathbb{R}_{+}^{K} \backslash\{0\}\right)^{n}$ be such that $a_{i}>b_{i}$ for all $i \in G_{1}$. Take $c \in\left(\mathbb{R}_{+}^{K} \backslash\{0\}\right)^{n}$ such that $a_{i}>c_{i}>b_{i}$ for all $i \in G_{1}$ and $c_{i}>\max \left\{a_{i}, b_{i}\right\}$ for all $i \in N \backslash G_{1}$. Since $a_{i}>c_{i}$ for all $i \in G_{1}$ and $c_{i}>a_{i}$ for all $i \in N \backslash G_{1}, a P c$ and by Weak Pareto, $c P b$, implying $a P b$. Therefore, for all $a, b \in\left(\mathbb{R}_{+}^{K} \backslash\{0\}\right)^{n}, a P b$ whenever $a_{i}>b_{i}$ for all $i \in G_{1}$.

Repeating this argument, one ultimately finds a subset containing a single individual $i_{0}$ such that for all $a, b \in\left(\mathbb{R}_{+}^{K} \backslash\{0\}\right)^{n}, a P b$ whenever $a_{i_{0}}>b_{i_{0}}$. That is the dictator.

Lemma 10 On the domain $\mathcal{R}^{n}$, if a social ordering function satisfies Weak Pareto and for every $K \subsetneq L, K \neq \emptyset$, there is a dictator over $\left(\mathbb{R}_{+}^{K} \backslash\{0\}\right)^{n}$, then there is a dictator over $\bar{X}$.

Proof of Lemma 10: First we prove that the same dictator rules over every $\left(\mathbb{R}_{+}^{K} \backslash\{0\}\right)^{n}$. 
Case 1: $\ell \geq 3$. Suppose not, with $i_{1}$ being a dictator over $\left(\mathbb{R}_{+}^{K} \backslash\{0\}\right)^{n}$ and $i_{2}$ over $\left(\mathbb{R}_{+}^{K^{\prime}} \backslash\{0\}\right)^{n}$. Let $K^{\prime \prime} \subsetneq L$ be such that $K \cap K^{\prime \prime} \neq \emptyset$ and $K^{\prime} \cap K^{\prime \prime} \neq \emptyset$, with $i_{3}$ the dictator over $\left(\mathbb{R}_{+}^{K^{\prime \prime}} \backslash\{0\}\right)^{n}$. Since $\left(\mathbb{R}_{+}^{K \cap K^{\prime \prime}} \backslash\{0\}\right)^{n} \subseteq\left(\mathbb{R}_{+}^{K} \backslash\{0\}\right)^{n} \cap\left(\mathbb{R}_{+}^{K^{\prime \prime}} \backslash\{0\}\right)^{n}$, both $i_{1}$ and $i_{3}$ must be dictators over $\left(\mathbb{R}_{+}^{K \cap K^{\prime \prime}} \backslash\{0\}\right)^{n}$, implying $i_{1}=i_{3}$. Similarly $i_{2}=i_{3}$, so that $i_{1}=i_{2}$. This proves that there is only one dictator $i_{0}$.

Case 2: $\ell=2$. By Lemma 9 , there is a dictator $i_{1}$ over $\left(\mathbb{R}_{++} \times\{0\}\right)^{n}$. Let $x, y \in$ $\left(\{0\} \times \mathbb{R}_{++}\right)^{n}$ be such that $x_{i_{1}}>y_{i_{1}}$. Then, there exist $z, w \in\left(\mathbb{R}_{++} \times\{0\}\right)^{n}$ such that for all $i \in N$, (i) if $x_{i}>y_{i}$, then $z_{i}>w_{i}$, and (ii) if $x_{i} \leq y_{i}$, then $z_{i}<w_{i}$. One can construct $R_{N}^{\prime} \in \mathcal{R}^{n}$ such that for all $i \in N$, either $x_{i} P_{i} z_{i} P_{i} w_{i} P_{i} y_{i}$ or $w_{i} P_{i} y_{i} R_{i} x_{i} P_{i} z_{i}$. In particular, $z_{i} P_{i_{1}} w_{i}$. Since $i_{1}$ is a dictator over $\left(\mathbb{R}_{++} \times\{0\}\right)^{n}$, one has $z P w$. By Weak Pareto, $x P z$ and $w P y$. By transitivity, $x P y$. By IIC and monotonicity of preferences, this ranking does not depend on individual preferences since $x, y \in\left(\{0\} \times \mathbb{R}_{++}\right)^{n}$. This means that $i_{1}$ is a dictator over $\left(\{0\} \times \mathbb{R}_{++}\right)^{n}$ as well.

Next, let $x, y \in \bar{X}$ and $R_{N} \in \mathcal{R}^{n}$ be such that $x_{i_{0}} P_{i_{0}} y_{i_{0}}$. Let $K, K^{\prime}$ be such that $x \in\left(\mathbb{R}_{+}^{K} \backslash\{0\}\right)^{n}, y \in\left(\mathbb{R}_{+}^{K^{\prime}} \backslash\{0\}\right)^{n}$. We have to prove that $x P y$. Let $z \in\left(\mathbb{R}_{+}^{K^{\prime}} \backslash\{0\}\right)^{n}$ be such that $x_{i_{0}} P_{i_{0}} z_{i_{0}} P_{i_{0}} y_{i_{0}}$ and for all $i \neq i_{0}, x_{i} P_{i} z_{i}$ (this is possible by continuity and the fact that $\left.x_{i} P_{i} 0\right)$. Because $i_{0}$ is a dictator over $\left(\mathbb{R}_{+}^{K^{\prime}} \backslash\{0\}\right)^{n}, z P y$, while $x P z$ by Weak Pareto. Therefore $x P y$ by transitivity.

This proves that there is a dictator over $\left(\mathcal{R}^{n}, \bar{X}\right)$.

Notice that $\mathcal{D}^{-} \cap \mathcal{R}^{n}=\mathcal{D}^{-}$and $X \cap \bar{X}=\bar{X}$, and for any $i, j \in N$ with $i \neq j$, there exist $R_{N}, R_{N}^{\prime} \in \mathcal{D}^{-}$and $x, y \in \bar{X}$ such that $x_{i} P_{i} y_{i}$ while $y_{j} P_{j} x_{j}$. If the dictator over $\left(\mathcal{D}^{-}, X\right)$ and the dictator over $\left(\mathcal{R}^{n}, \bar{X}\right)$ were different, it would be impossible to always adopt the strict preferences of both of them as strict social preferences. Hence, they must be the same agent. This completes the proof of Theorem 1.

We now show the tightness of Theorem 1, as announced in Section 5. For any 
$R_{N} \in \mathcal{D}^{+}=\mathcal{R}^{n} \backslash \mathcal{D}^{-}$, define the following correspondences:

$$
\begin{aligned}
X_{i}(k) & =\left\{x_{i} \in \mathbb{R}_{+}^{\ell} \mid \forall y_{i} \in \mathbb{R}_{+}^{L \backslash\{k\}}, x_{i} P_{i} y_{i}\right\} \\
I(k) & =\left\{i \in N \mid X_{i}(k) \neq \emptyset\right\}, \\
K(i) & =\{k \in L \mid i \in I(k)\} .
\end{aligned}
$$

Since $R_{N} \in \mathcal{D}^{+}, I(k) \neq \emptyset$ for all $k \in L$ and $\bigcup_{i \in N} K(i)=L$. Let us also prove that for all $i \in N$ and all $k \in K(i)$, the set $X_{i}(k)$ is closed (possibly empty), and if it is non-empty, it is equal to $u c\left(\bar{x}_{i}^{k} ; R_{i}\right)$ for some $\bar{x}_{i}^{k} \in \mathbb{R}_{+}^{\ell}$. Take any sequence $\left(z_{i}^{t}\right)_{t \in \mathbb{N}}$ in $X_{i}(k)$ which converges to some $z_{i} \in \mathbb{R}_{+}^{\ell}$. Assume that there is $y_{i} \in \mathbb{R}_{+}^{L \backslash\{k\}}$ such that $y_{i} R_{i} z_{i}$. Then, by strict monotonicity of preferences, there is $y_{i}^{\prime} \in \mathbb{R}_{+}^{L \backslash\{k\}}$ such that $y_{i}^{\prime} P_{i} z_{i}$. By continuity of preferences, there is $t \in \mathbb{N}$ such that $y_{i}^{\prime} P_{i} z_{i}^{t}$, contradicting the fact that $z_{i}^{t} \in X_{i}(k)$. This proves that $X_{i}(k)$ is closed. Moreover, for all $z_{i}, z_{i}^{\prime} \in \mathbb{R}_{+}^{\ell}$, if $z_{i}^{\prime} \in X_{i}(k)$ and $z_{i} R_{i} z_{i}^{\prime}$, then $z_{i} \in X_{i}(k)$. Take any $z_{i} \in X_{i}(k)$ and let

$$
\bar{x}_{i}^{k}=\min \left\{\alpha \in \mathbb{R}_{+} \mid \alpha z_{i} \in X_{i}(k)\right\} z_{i}
$$

By construction, $\bar{x}_{i}^{k} \in X_{i}(k)$, and for any $x_{i}^{\prime} \in \mathbb{R}_{+}^{\ell}$ such that $x_{i}^{\prime} R_{i} \bar{x}_{i}^{k}, x_{i}^{\prime} \in X_{i}(k)$. Conversely, for any $x_{i}^{\prime} \in X_{i}(k), x_{i}^{\prime} R_{i} \bar{x}_{i}^{k}$. For otherwise, if $\bar{x}_{i}^{k} P_{i} x_{i}^{\prime}$, there exists $\alpha^{\prime}<$ $\min \left\{\alpha \in \mathbb{R}_{+} \mid \alpha z_{i} \in X_{i}(k)\right\}$ such that $\alpha^{\prime} z_{i} P_{i} x_{i}^{\prime}$, implying that $\alpha^{\prime} z_{i} \in X_{i}(k)$, contradicting the fact that $\alpha^{\prime}<\min \left\{\alpha \in \mathbb{R}_{+} \mid \alpha z_{i} \in X_{i}(k)\right\}$.

Let

$$
u c_{i}^{k}= \begin{cases}X_{i}(k) & \text { if } i \in I(k) \\ \mathbb{R}_{+}^{\ell} & \text { if } i \notin I(k),\end{cases}
$$

and

$$
\overline{u c}_{i}=\bigcap_{k \in L} u c_{i}^{k} .
$$

By construction, for each $i \in N$, there is $\bar{x}_{i} \in \mathbb{R}_{+}^{\ell}$ such that $\overline{u c}_{i}=u c\left(\bar{x}_{i} ; R_{i}\right) .{ }^{14}$ As

\footnotetext{
${ }^{14}$ If $i \notin I(k)$ for all $k \in L$, then $\overline{u c}_{i}=u c\left(0 ; R_{i}\right)$.
} 
a consequence, for any $x \in \prod_{i \in N} \overline{u c}_{i}$ and any $y \notin \prod_{i \in N} \overline{u c}_{i}$, there is $i \in N$ such that $x_{i} R_{i} \bar{x}_{i} P_{i} y_{i}$, implying that $y$ does not Pareto-dominate $x$.

If $K(i) \neq \emptyset$ and $x_{i} \in \overline{u c}_{i}$, then for all $k \in K(i), x_{i} \in u c_{i}^{k}=X_{i}(k)$. Therefore, for all $y_{i} \in \mathbb{R}_{+}^{L \backslash\{k\}}, x_{i} P_{i} y_{i}$, so that necessarily $x_{i} \notin \mathbb{R}_{+}^{L \backslash\{k\}}$. In summary, one has $x_{i k}>0$ for all $x_{i} \in \overline{u c}_{i}$ and all $k \in K(i)$. Since $\bigcup_{i \in N} K(i)=L$, one then has $\sum_{i \in N} x_{i} \gg 0$ whenever $x_{i} \in \overline{u c}_{i}$ for all $i \in N$. Therefore, for all $K \subsetneq L$ and all $x \in\left(\mathbb{R}_{+}^{K}\right)^{n}, x \notin \prod_{i \in N} \overline{u c}_{i}$.

We can now define the social ordering $\Psi\left(R_{N}\right)$ as follows, for any $R_{N} \in \mathcal{R}^{n}$. Choose a reference bundle $x_{0} \in \mathbb{R}_{++}^{\ell}$. For all $x, y \in \mathbb{R}_{+}^{\ell n}, x R y$ if one of the following conditions holds:

(i) $R_{N} \in \mathcal{D}^{+}, x, y \in \prod_{i \in N} \overline{u c}_{i}$ and $\min _{i \in N} v_{R_{i}}\left(x_{i} ; x_{0}\right) \geq \min _{i \in N} v_{R_{i}}\left(y_{i} ; x_{0}\right)$, where $v_{R_{i}}\left(y_{i} ; x_{0}\right)$ is defined as in Section 5 ;

(ii) $R_{N} \in \mathcal{D}^{+}, x \in \prod_{i \in N} \overline{u c}_{i}$ and $y \notin \prod_{i \in N} \overline{u c}_{i}$;

(iii) $R_{N} \in \mathcal{D}^{+}, x, y \notin \prod_{i \in N} \overline{u c}_{i}$ and $x_{1} R_{1} y_{1}$;

(iv) $R_{N} \in \mathcal{D}^{-}$and $x_{1} R_{1} y_{1}$.

We check the properties of this social ordering function. Transitivity: For any $R_{N}$, this social ordering is transitive because, in the case of $R_{N} \in \mathcal{D}^{+}$, it partitions the set $\mathbb{R}_{+}^{\ell n}$ into two subsets, $\prod_{i \in N} \overline{u c}_{i}$ and its complement, ranks all allocations in $\prod_{i \in N} \overline{u c}_{i}$ above the others, and espouses transitive rankings within each subset. Weak Pareto: No allocation from the complement of $\prod_{i \in N} \overline{u c}_{i}$ can Pareto-dominate an allocation in $\prod_{i \in N} \overline{u c}_{i}$, and the specific rankings for $\prod_{i \in N} \overline{u c}_{i}$ and its complement satisfy Weak Pareto. IIC: For any for $K \subsetneq L$, if $x, y \in\left(\mathbb{R}_{+}^{K}\right)^{n}$ and if $R_{N}, R_{N}^{\prime} \in \mathcal{D}^{+}$, necessarily $x, y \notin \prod_{i \in N} \overline{u c}_{i}$, so that when $R_{N}$ and $R_{N}^{\prime}$ agree on $\mathbb{R}_{+}^{K}$, obviously $R_{1}$ and $R_{1}^{\prime}$ agree on $\{x, y\}$, and the corresponding social orderings do as well. A similar argument applies when $R_{N}, R_{N}^{\prime} \in \mathcal{D}^{-}$. When 
$R_{N} \in \mathcal{D}^{+}, R_{N}^{\prime} \in \mathcal{D}^{-}$, in both cases 1 dictates and the same argument applies again. Non-dictatorship: It is clear that, for every $R_{N} \in \mathcal{D}^{+}$, this social ordering function is not dictatorial over $\left(\left\{R_{N}\right\}, X\right)$.

We now provide an example of a social ordering function that is not dictatorial over $\left(\mathcal{R}^{n},\{x, y\}\right)$ for every pair $\{x, y\} \subseteq \mathbb{R}_{+}^{\ell n} \backslash \bar{X}$, except those such that $x_{i}>y_{i}$ for all $i \in N$ or $x_{i}<y_{i}$ for all $i \in N$, and those such that $x_{i}=y_{i}$ for some $i \in N$. Let $N^{*}\left(R_{N}\right) \subseteq N$ be the set of all $i \in N$ such that $R_{i}$ is strictly convex, when there are such agents, and let $N^{*}\left(R_{N}\right)=N$, otherwise. Modify the definition of the previous social ordering function by changing only part (i):

(i) $R_{N} \in \mathcal{D}^{+}, x, y \in \prod_{i \in N} \overline{u c}_{i}$ and $\min _{i \in N^{*}\left(R_{N}\right)} v_{R_{i}}\left(x_{i} ; x_{0}\right) \geq \min _{i \in N^{*}\left(R_{N}\right)} v_{R_{i}}\left(y_{i} ; x_{0}\right)$;

We only check non-dictatorship. Take a pair $x, y \in \mathbb{R}_{+}^{\ell n} \backslash \bar{X}$ such that it is not the case that $x_{i}>y_{i}$ for all $i \in N$ or $x_{i}<y_{i}$ for all $i \in N$, or $x_{i}=y_{i}$ for some $i \in N$. There is $R_{N} \in \mathcal{R}^{n}$ such that $x, y \in \prod_{i \in N} \overline{u c}_{i}$, every $R_{i}$ is strictly convex, and some agents prefer $x$ while all the others prefer $y$. Now, by slightly altering the convexity of $R_{N}$ without changing how every agent ranks $x_{i}, y_{i}$ and without upsetting $x, y \in \prod_{i \in N} \overline{u c}_{i}$, one can modify $N^{*}\left(R_{N}\right)$ at will and obtain either $x P y$ or $y P x$, so that no agent acts as a dictator.

Proof of Theorem 2: For dictatorship over $\left(\mathcal{D}^{-*}, X\right)$, the only part of the proof of Theorem 1 that needs substantial change is the proof of Lemma 1, which is now reformulated as follows. (The other lemmas used to prove Theorem 1 need to be changed in an obvious way to account for the person-specific nature of the $K_{i}$.)

Lemma 11 For each $i \in N$, let $K_{i} \subsetneq L$ be given. On the domain $\prod_{i \in N} \mathcal{R}_{K_{i}}$, if a social ordering function satisfies Weak Pareto and Individual Independence of Irrelevant Commodities, then it satisfies Weak Independence of Irrelevant Alternatives. 
Proof of Lemma 11. Let $R_{N}, R_{N}^{\prime} \in \prod_{i \in N} \mathcal{R}_{K_{i}}$ and $x, y \in X$ be such that for all $i \in N$, $R_{i}$ and $R_{i}^{\prime}$ agree on $\{x, y\}$, and for no $i \in N, x_{i} I_{i} y_{i}$. Assume that $x P y$. For each $i \in N$, define $M_{i}:=L \backslash K_{i} \neq \emptyset$. Let $R_{N}^{\prime \prime}$ be such that for each $i \in N$, every $K \subseteq L$ with $K \neq \emptyset$ is sufficient for $R_{i}^{\prime \prime}$ (for instance, this holds for a profile of linear preferences with positive normals), and $R_{i}$ and $R_{i}^{\prime \prime}$ agree on $\{x, y\}$.

Let $i \in N$ be given. Since $x, y>0$, by monotonicity of preferences we know that $y_{i} P_{i} 0$ and $x_{i} P_{i}^{\prime \prime} 0$. We can choose $z_{i}, w_{i}, z_{i}^{\prime}, w_{i}^{\prime} \in \mathbb{R}_{++}^{K_{i}}$ such that:

(i) if $x_{i} P_{i} y_{i}$ (and hence $x_{i} P_{i}^{\prime} y_{i}$ as well), then $z_{i} P_{i} x_{i} P_{i} y_{i} P_{i} w_{i}$ and $x_{i} P_{i}^{\prime \prime} z_{i}^{\prime} P_{i}^{\prime \prime} w_{i}^{\prime} P_{i}^{\prime \prime} y_{i}$,

(ii) if $y_{i} P_{i} x_{i}$ (and hence $y_{i} P_{i}^{\prime} x_{i}$ as well), then $y_{i} P_{i} w_{i} P_{i} z_{i} P_{i} x_{i}$ and $w_{i}^{\prime} P_{i}^{\prime \prime} y_{i} P_{i}^{\prime \prime} x_{i} P_{i}^{\prime \prime} z_{i}^{\prime}$, and

(iii) there are $\lambda_{i}, \lambda_{i}^{\prime} \in \mathbb{R}_{++}$such that $w_{i}=\lambda_{i} z_{i}, w_{i}^{\prime}=\lambda_{i}^{\prime} z_{i}^{\prime}$.

Next, choose $a_{i}, b_{i}, a_{i}^{\prime}, b_{i}^{\prime}, a_{i}^{\prime \prime}, b_{i}^{\prime \prime} \in \mathbb{R}_{++}^{M_{i}}$ such that:

(i) if $x_{i} P_{i} y_{i}$, then $a_{i}^{\prime}>a_{i}^{\prime \prime}>a_{i}>b_{i}>b_{i}^{\prime \prime}>b_{i}^{\prime}$, and

(ii) if $y_{i} P_{i} x_{i}$, then $b_{i}>b_{i}^{\prime \prime}>b_{i}^{\prime}>a_{i}^{\prime}>a_{i}^{\prime \prime}>a_{i}$, and

(iii) there are $\mu_{i}, \mu_{i}^{\prime} \in \mathbb{R}_{++}$such that $b_{i}=\mu_{i} a_{i}, b_{i}^{\prime}=\mu_{i}^{\prime} a_{i}^{\prime}$.

Let $R_{0} \in \mathcal{R}$ be an arbitrary preference relation on $\mathbb{R}_{+}^{\ell}$. As in the proof of Lemma 1 , we can construct a preference relation $R_{i}^{*} \in \mathcal{R}$ such that:

(i) $R_{i}^{*}$ and $R_{i}$ agree on $\mathbb{R}_{+}^{K_{i}}$.

(ii) $R_{i}^{*}$ and $R_{0}$ agree on $\mathbb{R}_{+}^{M_{i}}$.

(iii) $z_{i} I_{i}^{*} a_{i}$ and $w_{i} I_{i}^{*} b_{i}$.

Similarly, we construct $R_{i}^{\prime \prime *} \in \mathcal{R}$ such that:

(i) $R_{i}^{\prime \prime *}$ and $R_{i}^{\prime \prime}$ agree on $\mathbb{R}_{+}^{K_{i}}$.

(ii) $R_{i}^{\prime \prime *}$ and $R_{0}$ agree on $\mathbb{R}_{+}^{M_{i}}$.

(iii) $z_{i}^{\prime} I_{i}^{\prime \prime *} a_{i}^{\prime}$ and $w_{i}^{\prime} I_{i}^{\prime \prime *} b_{i}^{\prime}$.

Notice that $R_{i}^{*}$ and $R_{i}^{\prime \prime *}$ agree on $\mathbb{R}_{+}^{M_{i}}$ because they both agree with $R_{0}$. 
Having defined $z_{i}, w_{i}, z_{i}^{\prime}, w_{i}^{\prime} \in \mathbb{R}_{++}^{K_{i}}, a_{i}, b_{i}, a_{i}^{\prime}, b_{i}^{\prime}, a_{i}^{\prime \prime}, b_{i}^{\prime \prime} \in \mathbb{R}_{++}^{M_{i}}, R_{i}^{*}$ and $R_{i}^{\prime \prime *}$ for every $i \in N$, we obtain allocations $z, w, z^{\prime}, w^{\prime}, a, b, a^{\prime}, b^{\prime}, a^{\prime \prime}, b^{\prime \prime} \in \mathbb{R}_{+}^{\ell}$ and preference profiles $R_{N}^{*}:=\left(R_{1}^{*}, \ldots, R_{n}^{*}\right)$ and $R_{N}^{\prime \prime *}:=\left(R_{1}^{\prime \prime *}, \ldots, R_{n}^{\prime \prime *}\right)$. By construction, $R_{N}^{*}, R_{N}^{\prime \prime *} \in \prod_{i \in N} \mathcal{R}_{K_{i}}$.

By Weak Pareto, we have $z P x$ and $y P w$. By our supposition, $x P y$. Hence, transitivity of $P$ implies $z P w$. On the other hand, for every $i \in N$, since $a_{i}^{\prime \prime}>a_{i}, b_{i}>b_{i}^{\prime \prime}$, $z_{i} I_{i}^{*} a_{i}$ and $w_{i} I_{i}^{*} b_{i}$, strict monotonicity and transitivity of preferences imply $a_{i}^{\prime \prime} P_{i}^{*} z_{i}$ and $w_{i} P_{i}^{*} b_{i}^{\prime \prime}$. By Weak Pareto, $a^{\prime \prime} P^{*} z$ and $w P^{*} b^{\prime \prime}$. It follows from $z P w$ and Individual Independence of Irrelevant Commodities (IIIC) that $z P^{*} w$. By transitivity of $R^{*}$, we have $a^{\prime \prime} P^{*} b^{\prime \prime}$.

Similarly, by Weak Pareto, $x P^{\prime \prime} z^{\prime}$ and $w^{\prime} P^{\prime \prime} y$. By transitivity, strict monotonicity of preferences and Weak Pareto, we have $z^{\prime} P^{\prime \prime *} a^{\prime \prime}$ and $b^{\prime \prime} P^{\prime \prime *} w^{\prime}$. On the other hand, it follows from $a^{\prime \prime} P^{*} b^{\prime \prime}$ and IIIC that $a^{\prime \prime} P^{\prime \prime *} b^{\prime \prime}$. By transitivity of $P^{\prime \prime *}, z^{\prime} P^{\prime \prime *} w^{\prime}$. From IIIC (applied to $R_{N}^{\prime \prime *}$ and $R_{N}^{\prime \prime}$ ), we have $z^{\prime} P^{\prime \prime} w^{\prime}$. By transitivity, $x P^{\prime \prime} y$.

We have shown that $x P y \Rightarrow x P^{\prime \prime} y$. By symmetry, $x P^{\prime \prime} y \Rightarrow x P y$, and $y P x \Leftrightarrow$ $y P^{\prime \prime} x$. Hence, it also holds that $x I y \Leftrightarrow x I^{\prime \prime} y$. This means that $R$ and $R^{\prime \prime}$ agree on $\{x, y\}$.

By a similar reasoning, we can prove that $R^{\prime}$ and $R^{\prime \prime}$ agree on $\{x, y\}$. Therefore $R$ and $R^{\prime}$ agree on $\{x, y\}$.

The proof of dictatorship over $\left(\mathcal{R}^{n}, \overline{\bar{X}}\right)$ has exactly the same structure as the corresponding part of the proof of Th. 1. We first have the following lemma.

Lemma 12 On the domain $\mathcal{R}^{n}$, if a social ordering function satisfies Weak Pareto and Individual Independence of Irrelevant Commodities, then it satisfies Weak Independence of Irrelevant Alternatives restricted to allocations in $\overline{\bar{X}}$.

Proof of Lemma 12: Let $R_{N}, R_{N}^{\prime} \in \mathcal{R}^{n}$ and $x, y \in \overline{\bar{X}}$ be such that for all $i \in N, R_{i}$ and $R_{i}^{\prime}$ agree on $\{x, y\}$, and for no $i \in N, x_{i} I_{i} y_{i}$. Assume that $x P y$. 
For every $i \in N$, if $x_{i} P_{i} y_{i}$ then let $K_{i}=\left\{k \in L \mid x_{i k}>0\right\}$ and if $y_{i} P_{i} x_{i}$ then let $K_{i}=\left\{k \in L \mid y_{i k}>0\right\}$. Let $M_{i}=L \backslash K_{i}$. Since $x, y \in \overline{\bar{X}}$, we know that $K_{i}, M_{i} \neq \emptyset$.

Since $x_{i}, y_{i}>0$, by strict monotonicity of preferences we know that $y_{i} P_{i} 0$ and $x_{i} P_{i}^{\prime} 0$ for all $i$. We can choose $z, w, z^{\prime}, w^{\prime} \in \prod_{i \in N} \mathbb{R}_{+}^{K_{i}}$ such that for all $i \in N$,

(i) if $x_{i} P_{i} y_{i}$ (and hence $x_{i} P_{i}^{\prime} y_{i}$ as well), then $z_{i} P_{i} x_{i} P_{i} y_{i} P_{i} w_{i}$ and $x_{i} P_{i}^{\prime} z_{i}^{\prime} P_{i}^{\prime} w_{i}^{\prime} P_{i}^{\prime} y_{i}$,

(ii) if $y_{i} P_{i} x_{i}$ (and hence $y_{i} P_{i}^{\prime} x_{i}$ as well), then $y_{i} P_{i} w_{i} P_{i} z_{i} P_{i} x_{i}$ and $w_{i}^{\prime} P_{i}^{\prime} y_{i} P_{i}^{\prime} x_{i} P_{i}^{\prime} z_{i}^{\prime}$, and

(iii) there are $\lambda_{i}, \lambda_{i}^{\prime} \in \mathbb{R}_{++}$such that $w_{i}=\lambda_{i} z_{i}, w_{i}^{\prime}=\lambda_{i}^{\prime} z_{i}^{\prime}$.

Next, choose $a, b, a^{\prime}, b^{\prime}, a^{\prime \prime}, b^{\prime \prime} \in \prod_{i \in N} \mathbb{R}_{+}^{M_{i}}$ such that for all $i \in N$,

(i) if $x_{i} P_{i} y_{i}$, then $a_{i}^{\prime}>a_{i}^{\prime \prime}>a_{i}>b_{i}>b_{i}^{\prime \prime}>b_{i}^{\prime}$,

(ii) if $y_{i} P_{i} x_{i}$, then $b_{i}>b_{i}^{\prime \prime}>b_{i}^{\prime}>a_{i}^{\prime}>a_{i}^{\prime \prime}>a_{i}$, and

(iii) there are $\mu_{i}, \mu_{i}^{\prime} \in \mathbb{R}_{++}$such that $b_{i}=\mu_{i} a_{i}, b_{i}^{\prime}=\mu_{i}^{\prime} a_{i}^{\prime}$.

The rest of the argument is as in the proof of Lemma 11.

The rest of the proof applies without change for the case $\ell \geq 3$. When there are only two commodities, a more direct proof is necessary because there are no free triples. Indeed, in this case, in $\overline{\bar{X}}$ any individual bundle has only one commodity with a positive quantity, so that for any triple of bundles, there are at least two bundles with the same commodity. Strictly monotonic preferences rank this pair of bundles according to their respective quantity for this commodity.

Here is a proof for $\ell=2$. By Lemmas 9 and 10 and the fact that IIIC implies IIC, there is a dictator $i_{0}$ over $\bar{X}=\left(\mathbb{R}_{++} \times\{0\}\right)^{n} \cup\left(\{0\} \times \mathbb{R}_{++}\right)^{n}$.

Let $x, y \in \overline{\bar{X}}$ and $R_{N} \in \mathcal{R}^{n}$ be such that $x_{i_{0}} P_{i_{0}} y_{i_{0}}$ and for all $i \in N, x_{i} . y_{i}>$ 0 (meaning that the two bundles have a positive quantity for the same commodity). One can construct $z, w \in \bar{X}$ and $R_{N}^{\prime} \in \mathcal{R}^{n}$ such that for every $i \in N$, either $x_{i} P_{i} y_{i}$ and $x_{i} P_{i}^{\prime} z_{i} P_{i}^{\prime} w_{i} P_{i}^{\prime} y_{i}$, or $y_{i} R_{i} x_{i}$ and $w_{i} P_{i}^{\prime} y_{i} R_{i}^{\prime} x_{i} P_{i}^{\prime} z_{i}$. Because $i_{0}$ is a dictator over $\bar{X}$, 
$z P^{\prime} w$ and by Weak Pareto, $x P^{\prime} z$ and $w P^{\prime} y$. By transitivity, $x P^{\prime} y$ and by IIIC and monotonicity of preferences, $x P y$.

Let $x, y \in \overline{\bar{X}}$ and $R_{N} \in \mathcal{R}^{n}$ be such that $x_{i_{0}} P_{i_{0}} y_{i_{0}}$. One can construct $z \in \overline{\bar{X}}$ such that for every $i \in N, y_{i} . z_{i}>0$ and either $x_{i} P_{i} z_{i} P_{i} y_{i}$ or $y_{i} R_{i} x_{i} P_{i} z_{i}$. By the above property, one has $z P y$. By Weak Pareto, $x P z$, implying $x P y$. This shows that $i_{0}$ is a dictator over $\overline{\bar{X}}$.

Finally, by the same reasoning as the final step of the proof of Theorem 1 , the dictator over $\left(\mathcal{D}^{-*}, X\right)$ and the dictator over $\left(\mathcal{R}^{n}, \overline{\bar{X}}\right)$ must be the same agent. This completes the proof of Theorem 2 .

Again we briefly check the tightness of Theorem 2. For any $R_{N} \in \mathcal{D}^{+*}:=\mathcal{R}^{n} \backslash \mathcal{D}^{-*}$, there exists $i_{0}\left(R_{N}\right) \in N$ such that every $K \subseteq L$ with $K \neq \emptyset$ is indispensable for $R_{i_{0}\left(R_{N}\right)}$. We define the social ordering $\Psi\left(R_{N}\right)$ as follows. For all $x, y \in \mathbb{R}_{+}^{\ell n}, x R y$ if one of the following conditions holds:

(i) $R_{N} \in \mathcal{D}^{+*}, x_{i_{0}\left(R_{N}\right)}, y_{i_{0}\left(R_{N}\right)} \in \overline{u c}_{i_{0}\left(R_{N}\right)}$ and $x_{2} R_{2} y_{2}$;

(ii) $R_{N} \in \mathcal{D}^{+*}, x_{i_{0}\left(R_{N}\right)} \in \overline{u c}_{i_{0}\left(R_{N}\right)}$ and $y_{i_{0}\left(R_{N}\right)} \notin \overline{u c}_{i_{0}\left(R_{N}\right)}$;

(iii) $R_{N} \in \mathcal{D}^{+*}, x_{i_{0}\left(R_{N}\right)}, y_{i_{0}\left(R_{N}\right)} \notin \overline{u c}_{i_{0}\left(R_{N}\right)}$ and $x_{1} R_{1} y_{1}$;

(iv) $R_{N} \in \mathcal{D}^{-*}$ and $x_{1} R_{1} y_{1}$.

For every $R_{N} \in \mathcal{D}^{+*}$, this social ordering function is not dictatorial over $\left(\left\{R_{N}\right\}, X\right)$. A variant of this example in which agent 2 in (i) is replaced by the agent of smallest number (from 1 to $n$ ) in $N^{*}\left(R_{N}\right)$ would give non-dictatorship over $\left(\mathcal{R}^{n},\{x, y\}\right)$ for every pair $\{x, y\} \subseteq \mathbb{R}_{+}^{\ell n} \backslash \overline{\bar{X}}$, such that one does not have $x_{i}>y_{i}$ for all $i \in N$ or $x_{i}<y_{i}$ for all $i \in N$, or $x_{i}=y_{i}$ for some $i \in N$. 